This is an Open Access article, distributed under the terms of the Creative Commons Attribution licence (http://creativecommons.org/licenses/by/4.0/), which permits unrestricted re-use, distribution, and reproduction in any medium, provided the original work is properly cited.

\title{
Artificial intelligence control of a turbulent jet
}

\author{
Yu Zhou ${ }^{1, \dagger}$, Dewei Fan ${ }^{1}$, Bingfu Zhang ${ }^{1}$, Ruiying $\mathbf{L i}^{2}+$ \\ and Bernd R. Noack ${ }^{1,3, \dagger}$ \\ ${ }^{1}$ Institute for Turbulence-Noise-Vibration Interaction and Control, Harbin Institute of Technology, \\ Shenzhen 518055, PR China \\ ${ }^{2}$ Institut PPRIME, CNRS - Université de Poitiers - ISAE-ENSMA, \\ 86962 Futuroscope Chasseneuil, France \\ ${ }^{3}$ Institut für Strömungsmechanik und Technische Akustik (ISTA), Technische Universität Berlin, \\ Müller-Breslau-Straße 8, D-10623 Berlin, Germany
}

(Received 23 October 2019; revised 8 May 2020; accepted 9 May 2020)

An artificial intelligence (AI) control system is developed to maximize the mixing rate of a turbulent jet. This system comprises of six independently operated unsteady minijet actuators, two hot-wire sensors placed in the jet and genetic programming for the unsupervised learning of a near-optimal control law. The ansatz of this law includes multi-frequency open-loop forcing, sensor feedback and nonlinear combinations thereof. Mixing performance is quantified by the decay rate of the centreline mean velocity of the jet. Intriguingly, the learning process of AI control discovers the classical forcings, i.e. axisymmetric, helical and flapping achievable from conventional control techniques, one by one in the order of increased performance, and finally converges to a hitherto unexplored forcing. Careful examination of the control landscape unveils typical control laws, generated in the learning process, and their evolutions. The best AI forcing produces a complex turbulent flow structure that is characterized by periodically generated mushroom structures, helical motion and an oscillating jet column, all enhancing the mixing rate and vastly outperforming others. Being never reported before, this flow structure is examined in various aspects, including the velocity spectra, mean and fluctuating velocity fields and their downstream evolution, and flow visualization images in three orthogonal planes, all compared with other classical flow structures. Along with the knowledge of the minijet-produced flow and its effect on the initial condition of the main jet, these aspects cast valuable insight into the physics behind the highly effective mixing of this newly found flow structure. The results point to the great potential of AI in conquering the vast opportunity space of control laws for many actuators and sensors and in optimizing turbulence.

Key words: mixing enhancement, jets, turbulence control

$†$ Email addresses for correspondence: yuzhou@hit.edu.cn, bernd.noack@hit.edu.cn $\ddagger$ Present address: Renault Group 1, Avenue du Golf 78280, Guyancourt, France. 


\section{Introduction}

The turbulent jet is one of the classical shear flows discussed in virtually every textbook. Its control finds important industrial applications, including dilution jets in combustors, fuel injection of combustion engines, noise mitigation of sub- and supersonic jets for civil and military aircrafts, thrust augmenting ejectors, thrust vector control, etc. The key to control entrainment and mixing processes in a turbulent jet is, as in other shear layers, to manipulate the coherent motions. When a jet issues from a round nozzle, a free shear layer is formed from the nozzle lip and develops downstream. A Kelvin-Helmholtz instability (Ho \& Huerre 1984) inherent in the shear layer rapidly grows, resulting in the formation of axisymmetric ring vortices. The vortices, along with their subsequent interaction (e.g. merging and breakdown), dominate the shear-layer growth and entrainment (Crow \& Champagne 1971). Shortly downstream of the nozzle exit, three-dimensionality becomes an important feature of the flow structure; streamwise vorticity contributes predominantly to the entrainment of fluid from the surroundings (Liepmann \& Gharib 1992). These motions, formed near the nozzle exit, are featured by a wide range of scales, varying convection velocity and a rich set of three-dimensional patterns (Garnaud et al. 2013); they are sensitive to initial conditions (e.g. the turbulence level, boundary layer thickness, nozzle geometry) and external periodic disturbances (Vlasov \& Ginevskii 1973), thus, highly susceptible for control.

Jet control can be active or passive. Passive control involves a change in geometry such as tabs (e.g. Bradbury \& Khadem 1975), non-circular nozzles (e.g. Husain \& Hussain 1983) and chevron nozzles (e.g. Alkislar, Krothapalli \& Butler 2007). Although often highly effective, passive techniques are characterized by permanent fixtures. Once mounted, tabs are difficult to be relocated. Likewise, it is impractical for any engineering application to implement frequently non-circular nozzle geometry alteration due to cost and physical constraints. Furthermore, there are other penalties, e.g. thrust loss and drag. Active control requires the input of external power, e.g. acoustic excitation (e.g. Zaman \& Hussain 1981), piezo-electric actuators (e.g. Wiltse \& Glezer 1993), plasma actuators (e.g. Samimy et al. 2007), synthetic jet (e.g. Tamburello \& Amitay 2007), flip-flop jets (e.g. Raman, Hailye \& Rice 1993) and steady/unsteady minijets (Zhou et al. 2012; Yang \& Zhou 2016). The active method has potential to achieve more flexible and drastic flow modifications, which is a great advantage over the passive (e.g. Zaman, Reeder \& Samimy 1994; Longmire \& Duong 1996; Reeder \& Samimy 1996).

Many active control studies of turbulent jets involve the open-loop periodic forcing of a prespecified form, e.g. axisymmetric, flapping or helical forcing. Broze \& Hussain (1994) deployed four speakers upstream of the nozzle to add a longitudinal component of perturbation to the mean flow. The acoustic source produced axisymmetric forcing which was found to amplify vortex ring structures and subsequent vortex pairing. Koch et al. (1989) generated helical forcing on a turbulent round air jet using four speakers, each being $90^{\circ}$ out of phase with the adjacent speaker. Yang et al. (2016) used two unsteady radial minijets separated by $60^{\circ}$ or $120^{\circ}$ to produce a flapping jet column, which enhanced greatly jet mixing. The combination of individual forcings is also investigated. Juvet (1987) optimized experimentally the combinations of axisymmetric axial and helical forcing to augment mixing. The axial excitation was produced by a loudspeaker placed upstream of the jet and perpendicularly to the centreline. The helical excitation was generated by four external loudspeakers. Their acoustic excitations were directed via waveguides at an angle around the jet exit lip to the shear layer where the flow is most sensitive to acoustic forcing. 
While the axial excitation led to the early formation of large-scale vortices, the helical caused the shear layer to roll up into staggered vortex structures. This combined excitation generated a bifurcating jet with a much larger spreading angle than the single excitation when the frequency ratio of the axial to that of the helical excitation was equal to 2 (Reynolds et al. 2003). Three-dimensional direct numerical simulation of a turbulent jet by Hilgers \& Boersma (2001) demonstrated that the superposition of two counter-rotating helical modes of the same excitation frequency $f_{e}$ and one axial excitation of $2 f_{e}$ produced a bifurcating jet whose centreline mean velocity and scalar concentration decayed faster than those of the counter-rotating helical actuation alone.

Tyliszczak \& Geurts (2015) and Tyliszczak (2018) simulated highly mixed multi-armed bifurcating jets using axial and helical excitations. However, it would be very difficult or time-consuming for conventional active controls to find the globally optimal solution for the combined excitations where many control parameters are generally involved. For instance, the control optimization of a turbulent jet has so far typically involved up to two control parameters, such as the actuation amplitude and frequency. Then, the optimization of combined modes, like axisymmetric forcing and flapping forcing, may involve at least four independent control parameters, i.e. the amplitude and frequency of each mode (e.g. Hilgers \& Boersma 2001). The search for its optimal solution is then already a challenge. If the control parameters for each mode is increased to three or four such as the amplitude, frequency, duty cycle and diameter ratio of an unsteady jet (e.g. Perumal \& Zhou 2018) or multiple independent actuators are deployed, the search for the globally optimal solution of the combined modes would be a daunting task. Koumoutsakos, Freund \& Parekh (2001) and Hilgers \& Boersma (2001) have pioneered the jet mixing optimization with three and four actuation parameters using Rechenberg's (1973) evolutionary strategy.

Model-based control comes, if doable, with the deep understanding of actuation dynamics, regardless of open or closed loops. In simulations, the linear dynamics can be accurately resolved by discretized Navier-Stokes $(\mathrm{N}-\mathrm{S})$ equations (Kim \& Bewley 2007; Sipp et al. 2010). In experiments, linear stochastic estimation (Tinney et al. 2006) has been successfully applied to resolve the flow physics from measurement signals and PIV measurements. The linearized $\mathrm{N}-\mathrm{S}$ dynamics can be encapsulated in reduced-order models employing several dominant non-normal global stability eigenmodes. The downstream evolution of wavepackets can be real-time estimated in a high-Reynolds-number turbulent jet thanks to the development of transfer functions based on the parabolized stability equations (Sasaki et al. 2017). So can the closed-loop control of fluctuations in a low-Reynolds-number shear layer (Sasaki et al. 2018). These control-oriented models have significantly contributed to the understanding of the manipulated jet dynamics.

Model-free approaches may yield performance benefits from nonlinear dynamics which is too complex for control-oriented models. A new model-free self-learning approach for general nonlinear control laws has been developed by Dracopoulos (1997) for commanding satellite motion and was rediscovered in fluid mechanics as machine learning control or MLC (Gautier et al. 2015). A review of dozens of MLC experiments and simulations is provided by Noack (2019). The first MLC experiment was set to enhancing shear-layer mixing with 96 jet actuators driven in unison and 25 hot-wire sensors for feedback control (Parezanović et al. 2016). The optimization of shear-layer mixing resulted in destabilizing phasor control, i.e. the feedback excitation of the dominant frequency. The control enhanced and synchronized downstream large-scale vortices with a frequency of one sixth of the initial Kelvin-Helmholtz (K-H) instability. Li et al. (2017) deployed four Coanda jet actuators placed at the 
trailing edge of an Ahmed body and 12 pressure sensors at the back side, achieving a drag reduction by $22 \%$ (where about $10 \%$ was attributed to the Coanda effect) when the excitation frequency was much higher than the predominant and even the shear-layer frequencies in the wake. Using an unsteady single jet actuator driven by an online PIV-based sensing, Gautier et al. (2015) cut short the reattachment length of flow over a backward-facing step. They observed surprisingly the enhancement of a low-frequency flapping mode, instead of the excitement of the dominant $\mathrm{K}-\mathrm{H}$ vortex shedding. Machine learning control has matched with or outperformed existing control strategies and solved the combined task of picking the nonlinear mechanisms for performance optimization and selecting the best sensors. These model-free control studies show that the actuation mechanism can be very difficult to anticipate, thus implying a challenge to any model-based control.

It is worth pointing out that MLC has never been applied to multiple independently operated actuators, resembling a distributed actuation, in experiments so far. Machine learning control laws, previously developed, have been of small to moderate complexity, e.g. the phasor control, threshold-level based control, periodic or two-frequency forcing (Duriez, Brunton \& Noack 2016), as the actuators are typically driven by a single actuation command. Indeed, the use of independent actuators may increase dramatically the level of control complexity. For example, assume that one unsteady minijet, used to maximize jet mixing, involves three parameters, i.e. the actuation frequency $f_{a}$, velocity $U_{a}$ and duty cycle $\alpha$. Then, if the number is increased to up to say six independent minijets spatially distributed around the main jet, the independent control parameters will be tremendously increased. Then one naturally wonders what the globally optimal solution of the problem is and whether an AI control technique could be developed to find this solution. Furthermore, what turbulent flow structure might this global optimal solution or forcing produce?

This experimental work aims to address the issues raised above and to optimize jet entrainment/mixing with six independently unsteady minijets placed upstream of the nozzle exit, extending the MLC jet control using a single unsteady minijet (Wu et al. 2018a). The manuscript is organized as follows. The experimental setup and minijet-produced flow, along with its effect on the jet initial conditions, are described in $\S \S 2$ and 3, respectively. In $\S \S 4,5$ and 6 we respectively describe the AI control system developed, the outcome of the AI-based learning and the resulting turbulent flow structures. The work is concluded in $\S 7$.

\section{Experimental details}

\subsection{Jet facility}

Experiments were conducted in a round air jet facility, as schematically shown in figure 1. The facility was placed in an air-conditioned laboratory where the room temperature remains constant within $\pm 0.5^{\circ} \mathrm{C}$, centrally deployed in an area of approximately $2.5 \mathrm{~m}$ in width and $2 \mathrm{~m}$ in height, enclosed by fabric walls. In order to minimize the effects of the wall on the jet, the nozzle exit is $4.0 \mathrm{~m}$ away from the fabric partition wall and the distance is well over 70 times the jet exit diameter required for neglecting the wall effects (Malmstrom et al. 1997). As the jet is highly sensitive to background noise, careful measures are taken to avoid any external interference to airflow.

The compressed air of the round jet comes from a constant 5 bar gauge pressure, mixed with seeding particles in the mixing chamber in the case of the particle image 
(a)

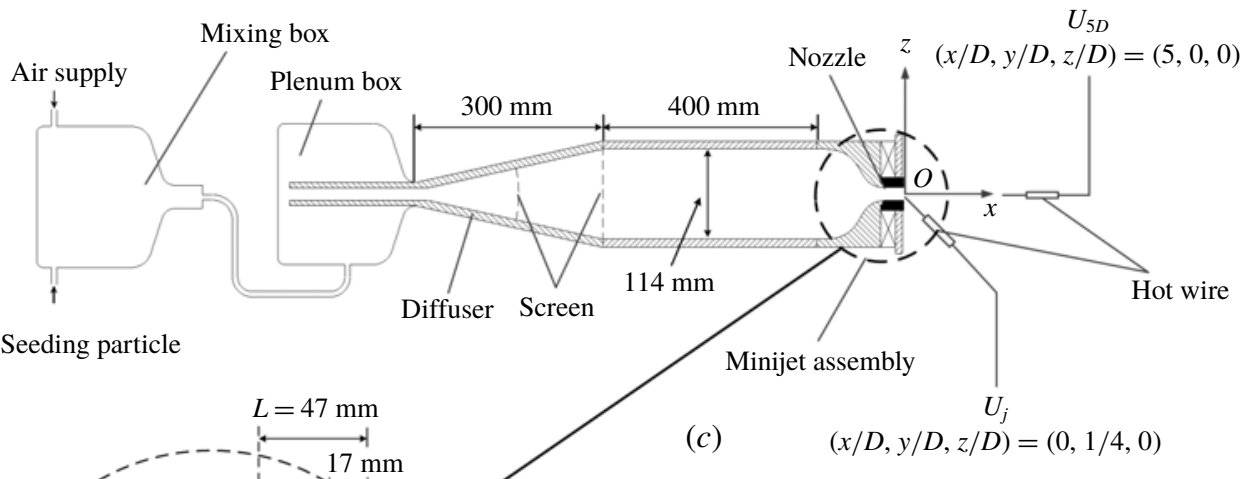

(b)
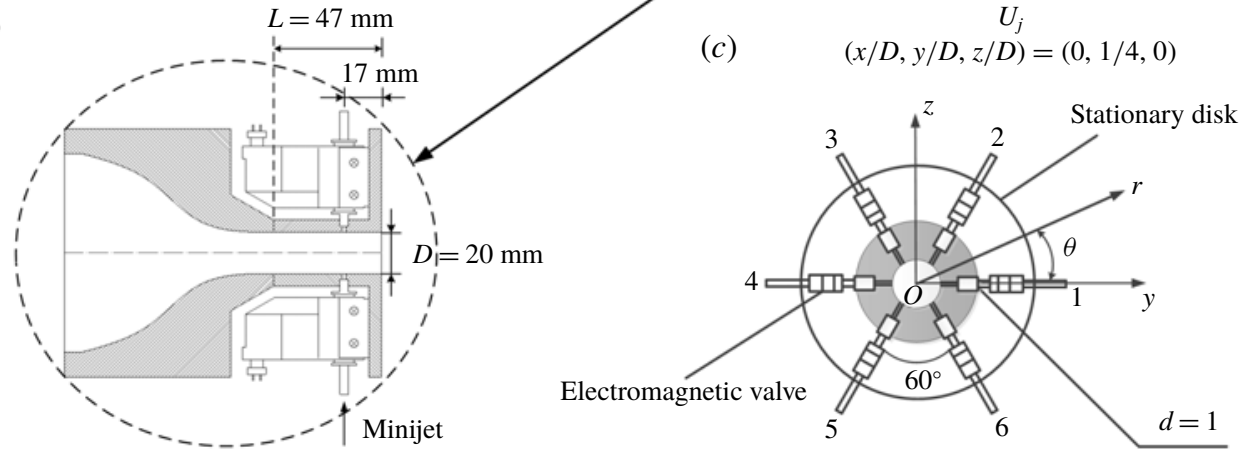

FIGURE 1. Sketch of the experimental setup: (a) main jet facility; $(b)$ minijet assembly; (c) minijet arrangement.

velocimetry (PIV) or flow visualization measurements, and then enters into a plenum chamber, composed of a $300 \mathrm{~mm}$ long diffuser of $15^{\circ}$ in half-angle and a $400 \mathrm{~mm}$ long cylindrical settling chamber with an inner diameter of $114 \mathrm{~mm}$. The flow passes two screens before entering the smooth contraction nozzle (Perumal \& Zhou 2018), which is extended by a $47 \mathrm{~mm}$ long smooth tube of the same diameter as the nozzle exit $D(=20 \mathrm{~mm})$. The Reynolds number $R e_{D}=\bar{U}_{j} D / v$ of the main jet is fixed at 8000 , where $U_{j}$ is the centreline velocity measured at the nozzle exit, the overbar denotes time-averaging and $v$ is the kinematic viscosity of air. A Cartesian coordinate system $(x, y, z)$ is defined in figure $1(a, c)$, with its origin at the centre of the jet exit and the $x$-axis pointing in the direction of flow. Measurements were conducted in the $x-z, x-y$ and $y-z$ planes of the main jet. The instantaneous and fluctuating velocities in the $x, y$ and $z$ directions are denoted by $(U, V, W)$ and $(u, v, w)$, respectively.

Six unsteady control minijets issued from orifices with a diameter of $1 \mathrm{~mm}$ are equidistantly placed around the extension tube at $x_{i}=-0.85 D, y_{i}=(D / 2) \cos \theta_{i}$, $z i=(D / 2) \theta_{i}$, where $\theta_{i}=(i-1) 2 \pi / 6, i=1,2, \ldots, 6$ (figure $\left.1 b, c\right)$. Their mass flow rate is determined by a flow-limiting valve and monitored by a mass flow meter, with a measurement uncertainty of $1 \%$, and the frequencies and duty cycles are independently controlled by individual electromagnetic valves that are operated in an ON/OFF mode. The maximum operating frequency of the valves is $500 \mathrm{~Hz}$, exceeding three times the preferred-mode frequency, $f_{0}=135 \mathrm{~Hz}$, of the unforced jet, the corresponding Strouhal number being $S_{t}=f_{0} D / \bar{U}_{j}=0.45$, where $f_{0}$ is obtained from the power spectral density function $E_{u}$ of the streamwise fluctuating velocity $u$ measured in the absence of control (Yang \& Zhou 2016). 


\subsection{Flow measurements}

The fluctuating flow velocities are monitored by two tungsten wire sensors of $5 \mu \mathrm{m}$ in diameter, operated on a constant temperature circuit (Dantec streamline) at an overheat ratio of 0.6 , one placed at $(x / D, y / D, z / D)=(0,1 / 4,0)$ and the other at $(x / D, y / D, z / D)=(5,0,0)$. The time-averaged velocity at the latter position is denoted by $U_{5 D}$. This choice is based on the following considerations. Firstly, Zhou et al. (2012) demonstrated that the decay rate of the centreline mean velocity of jet defined by $K=\left(\bar{U}_{j}-\bar{U}_{5 D}\right) / \bar{U}_{j}$ is correlated approximately linearly with an equivalent jet half-width $R_{e q}=\left[R_{H} R_{V}\right]^{0.5}$, where $R_{H}$ and $R_{V}$ are the jet half-widths in two orthogonal planes, implying that $K$ is directly connected to the entrainment rate of the manipulated jet. Secondly, Fan et al. (2017) found that the difference $\triangle K$ between the $K$ values with and without control reaches the maximum at $x / D \approx 5$, that is, the centreline mean streamwise velocity measured at $x / D=5$ is most sensitive to the change in the control parameters. Finally, the variation in $K$ is almost linear from $x / D=0$ to $x / D \approx 7$ under control (figure 7, Fan et al. (2017)), that is, a single value of $K$ may be used to describe reasonably well the jet decay rate in the near field under control. Both hot wires are calibrated at the jet exit using a pitot-static tube connected to a micromanometer (Furness Controls FCO510). The cutoff and sampling frequencies are $3 \mathrm{kHz}$ and $6 \mathrm{kHz}$ for open-loop control experiments, respectively. The experimental uncertainty of the hot-wire measurement is estimated to be less than $2 \%$.

A planar high-speed PIV system, with a high-speed camera (Dantec speed sensor $90 \mathrm{C} 10,2056 \times 2056$ pixels resolution) and a pulsed laser source (Litron LDY304PIV, Nd: YLF, $30 \mathrm{~mJ}$ pulse ${ }^{-1}$ ), is deployed for velocity field measurements in the $x-z, x-y$ and $y-z$ planes. An oil droplet generator (TSI MCM-30) is used to generate fog from olive oil with an averaged particle size of $1 \mu \mathrm{m}$ for flow seeding. Flow illumination is provided by a laser sheet of $1 \mathrm{~mm}$ in thickness generated by the pulsed laser via a cylindrical lens. For velocity measurements in the $x-z$ and $x-y$ planes, the captured image covers the area of $x / D \in[0,6]$ and $y / D, z / D \in[-2,2]$. The longitudinal and lateral image magnifications are identical, $0.09 \mathrm{~mm}$ per pixel. The time interval between two consecutive images is presently chosen to be $25 \mu \mathrm{s}$, which is found to yield satisfactory results. There are $253 \times 253$ velocity vectors, the same for the two planes. A total of 200 pairs of flow images are captured at a sampling rate of $405 \mathrm{~Hz}$ for each set of PIV data. In post-processing, a built-in adaptive correlation function of the flow map processor (PIV 2001 type) is applied with an interrogation window of $32 \times 32$ pixels and a $75 \%$ overlap along both directions.

The same PIV system is used for flow visualization in the three orthogonal planes. So are the seeding particles, though their concentration is higher than in the PIV measurements to provide a clear picture for the flow structure. The captured images cover the area of $x / D \in[0,6]$ and $y / D$ or $z / D \in[-2,2]$ in the $x-y$ and $x-z$ planes and the area of $y / D=z / D \in[-2,2]$ at $x / D=0.25$ in the $y-z$ plane.

\subsection{Real-time system}

A national instrument PXIe-6356 multifunction I/O device, connected to a computer, is used in experiments to generate the real-time control command at a sampling rate of $F_{r f}=1 \mathrm{kHz}$. A LabVIEW real-time module is used to execute the program. Sensor data acquisition and control command generation for the AI control experiments are operated under the same sampling frequency of $1 \mathrm{kHz}$. It has been confirmed that the $\mathrm{ON} / \mathrm{OFF}$ command lasts at least $1 \mathrm{~ms}$ to ensure the actuators work effectively. The available $f_{a}$ can be derived from $f_{a}=F_{r f} / N_{s p}$, where $N_{s p}$ is the number of sampling 


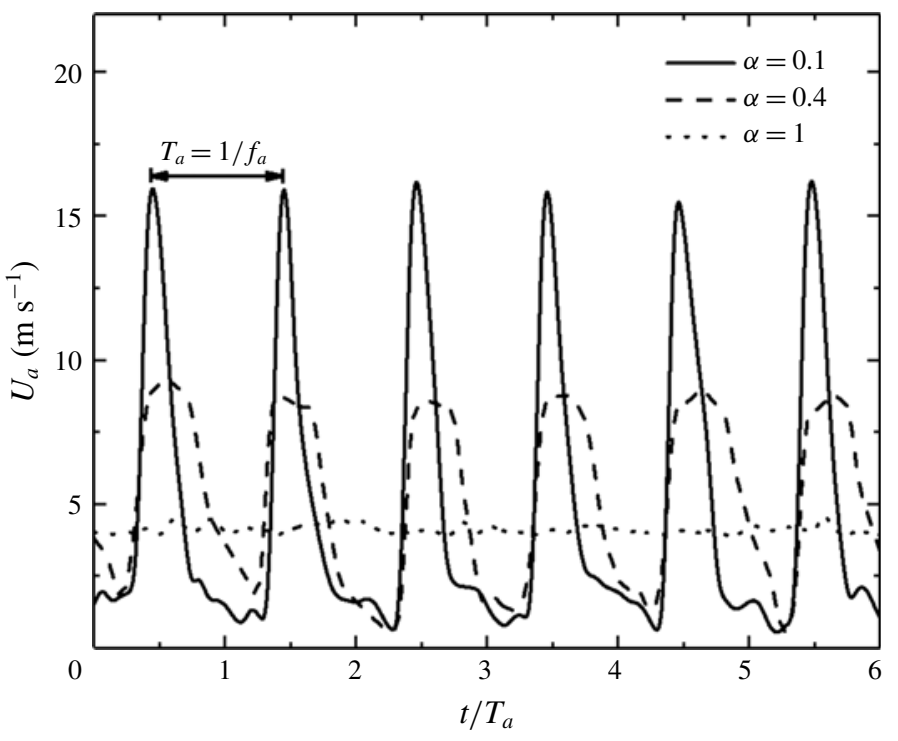

FIGURE 2. Time histories of minijet injection velocity $U_{a}$ at duty cycles $\alpha=0.1,0.4$ and 1 (see legend) in the absence of the main jet measured at $(x / D, y / D, z / D)=$ $(-0.85,-0.35,0)$ for $C_{m}=1.2 \%, f_{a} / f_{0}=0.5 . \overline{U_{j}}=0$.

points in one period $1 / f_{a}$. The working frequency range of actuators $[0,500 \mathrm{~Hz}]$ imposes a minimum value for $N_{s p}$, i.e. $N_{s p} \geqslant 2$. For a given frequency, $\alpha$ can be deduced from $m / N_{s p}, m=1, \ldots, N_{s p}-1$. The $m$ range ensures a response time of $1 \mathrm{~ms}$ for the effective working of the actuators, which is adequate as the maximum sampling rate $F_{r f}$ is $1 \mathrm{kHz}$ due to the limitation of hardware. Thus, the number of possible duty cycles $N_{\alpha}$ for a given $f_{a}$ is $N_{\alpha}=N_{s p}-1=F_{r f} / f_{a}-1$, which increases with $F_{r f}$ and decreases with $f_{a}$. This process is similar to the one used by Li et al. (2017) and Wu et al. (2018a).

\section{Minijet actuation}

\subsection{Minijet-produced flow}

It is important to document the flow produced by a minijet and the effect of minijets on the initial condition of the main jet. This information is crucial for understanding physically the manipulated jet. The instantaneous velocity $U_{a}$ of a single radial minijet is first examined in the absence of the main jet. A hot wire is placed $17 \mathrm{~mm}$ or $x / D=$ -0.85 upstream of the main jet exit and $3 \mathrm{~mm}$ radially from the exit of minijet 1 (figure $1 c$ ). The hot wire is oriented normal to the minijet axis - recording the signal $U_{a}$, which changes with $\alpha$ (figure 2). For $\alpha=0.1, U_{a}$ displays sharp peaks which are periodic and clearly separated. But these peaks are less pronounced at $\alpha=0.4$. The signal $U_{a}$ is almost steady at $\alpha=1$, though showing a small variation, as observed by Johari, Pachecotougas \& Hermanson (1999). Apparently, a small $\alpha$ produces a large instantaneous velocity, implying a large penetration depth into the main jet.

Consider the simultaneous injection of minijets 1 and 4 (figure 1c) without the main jet. Two hot wires are placed perpendicularly to the $x-y$ plane at $x / D=-0.85$ and $3 \mathrm{~mm}$ from each of the corresponding measured minijet exit. The two minijets 


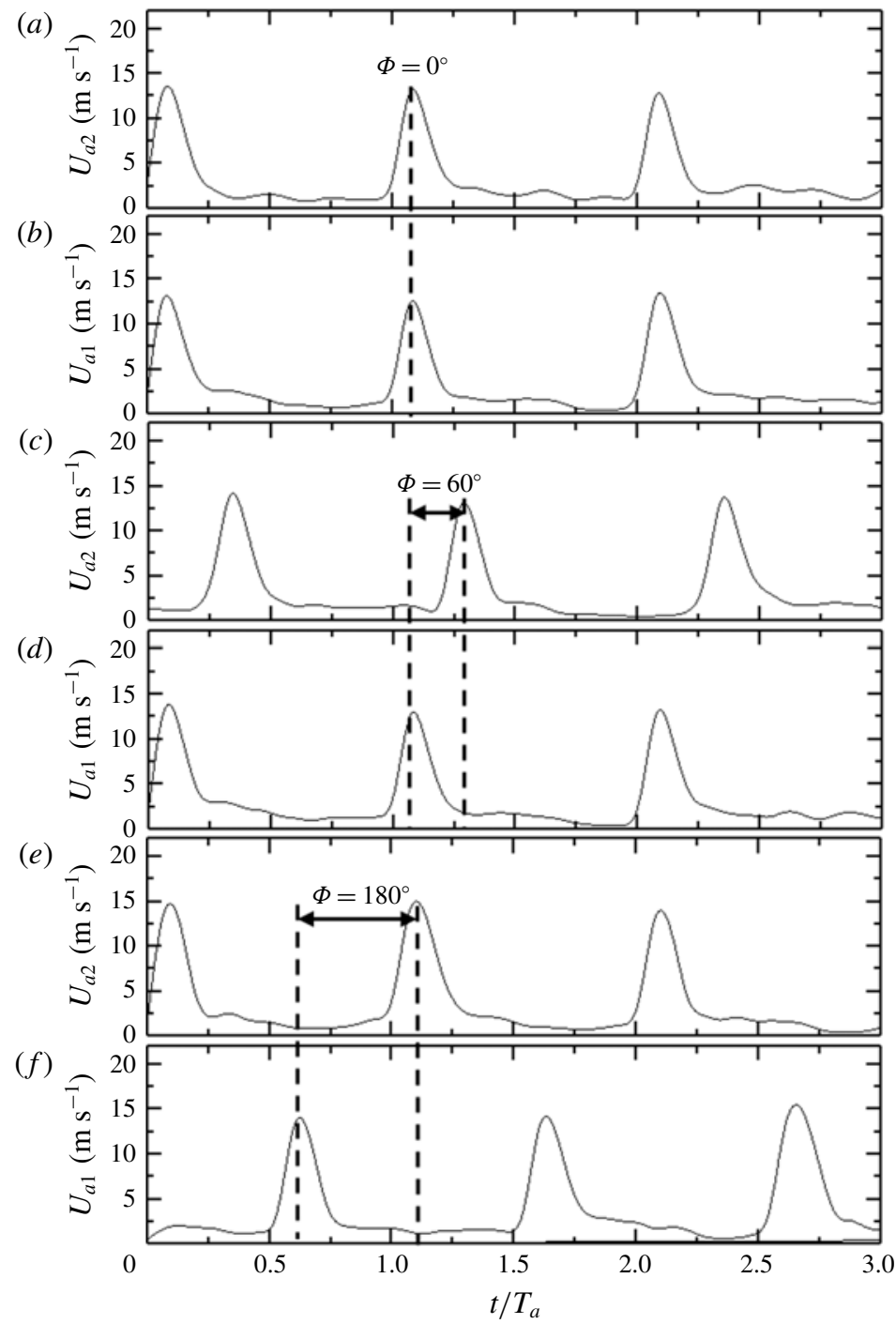

FIgURE 3. Time histories of two minijet injection velocity signals $U_{a 1}, U_{a 2}$ measured simultaneously at $(x / D, y / D, z / D)=(-0.85, \pm 0.35,0)$ for $C_{m}=1.2 \%, f_{a} / f_{0}=0.5$ and $\alpha=0.1$. There is a phase difference $\Phi$ between two minijets control signals: $(a-b) \Phi=0$, $(c-d) \Phi=60^{\circ},(e-f) \Phi=180^{\circ} . \bar{U}_{j}=0$.

are injected with a phase shift $\Phi$, which may be varied by changing the phase shift between the two square wave signals of input voltages. At $\Phi=0^{\circ}$, the $U_{a 1}$ signal exhibits a very sharp peak value, with a magnitude of close to 0 at the off-state of the minijet and about 13 at the on-state (figure $3 a$ ). Note that, even after the electromagnetic valve is closed, there may be some fluid injecting into the main jet (Sailor, Rohli \& Fu 1999). A similar observation can be made for $\Phi=60^{\circ}$ and $180^{\circ}$ (figure $3 b, c$ ). The characteristics of $U_{a 2}$ resemble those of $U_{a 1}$, regardless of the $\Phi$ value. It may be inferred that each of the minijets does not depend on $\Phi$ and is rather independent of each other. 

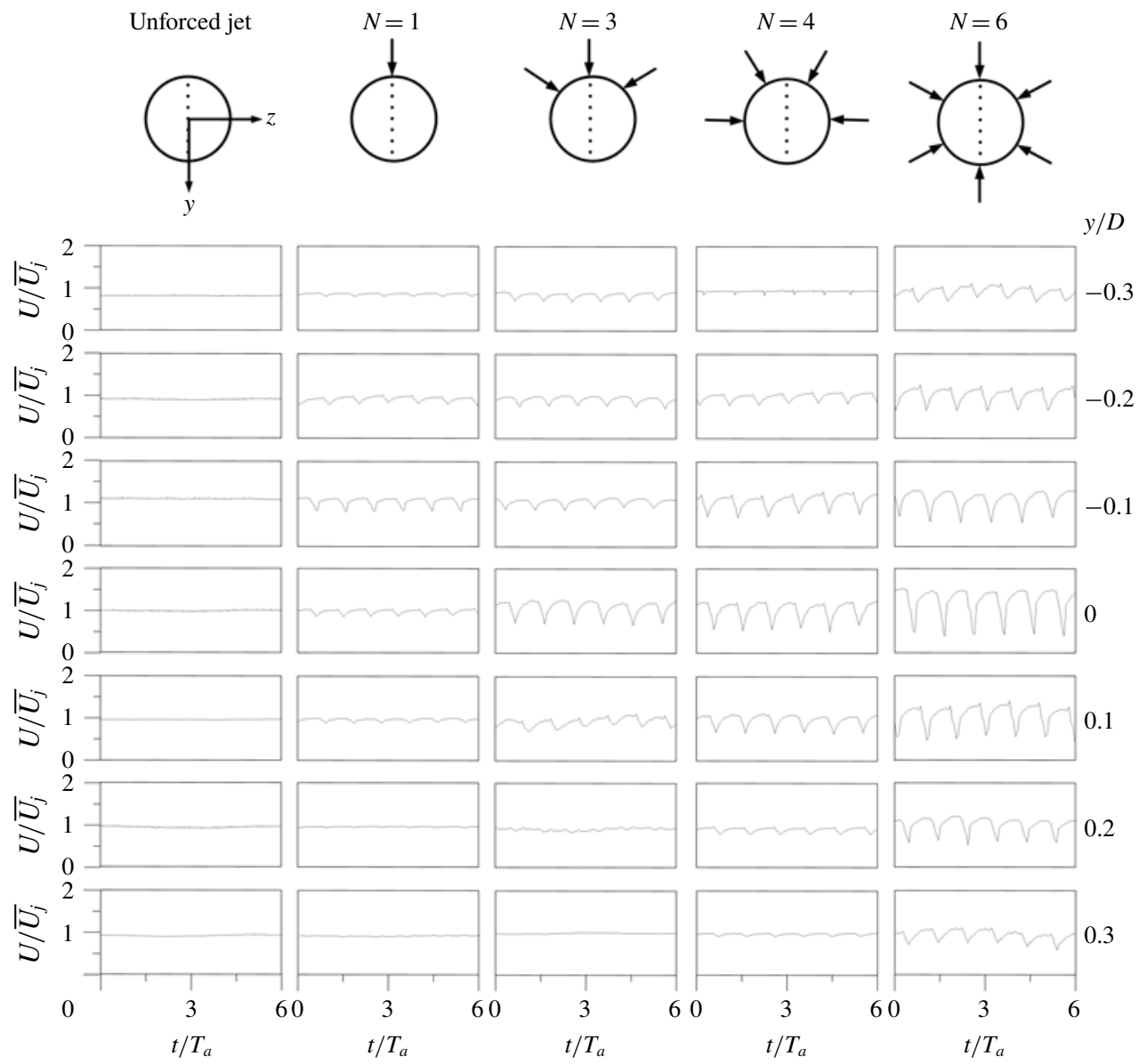

FIGURE 4. Typical hot-wire signals of instantaneous streamwise velocity $U / \overline{U_{j}}$ along $x-y$ plane at $x / D=0.05$ for minijet numbers $N=1,3,4$ and $6\left(f_{a} / f_{0}=0.5, \alpha=0.15, C_{m}=\right.$ $1.2 \%)$. The same scale is applied for all signals. The dots within the circle represent the hot-wire measurement points.

\subsection{Penetration depth and minijet number}

The penetration depth of control jets may have a pronounced impact upon jet mixing (Davis 1982). Thus, its influence on the main jet is examined for various minijet numbers and configurations with given $\bar{U}_{j}$ and control parameters, i.e. $\alpha, f_{a} / f_{0}$ and mass flow ratio $C_{m}=m_{\text {mini }} / m_{j}$, where $m_{\text {mini }}$ and $m_{j}$ are the mass flow rates of a single minijet and main jet, respectively. The minijet penetration depth could be approximately estimated from the $U$ signals along the $y$-direction, measured at $x / D=0.05$ using a hot wire placed perpendicularly to the $x-y$ plane, as shown in figure 4 , where the scale of the abscissa or ordinate is made the same for all cases to facilitate comparison. The $U$ signals are essentially constant throughout the range of $y / D \in[-0.3,0.3]$ for the unforced jet. The periodic fluctuations of $U$ appear at $y / D=-0.3$ for one minijet injection $(N=1)$, and its magnitude grows first and then retreats with increasing $y / D$. The fluctuations remain discernible at $y / D=-0.1$. Note that the minijet is issued along the $y$-direction. Beyond $y / D=-0.1$, the velocity 
fluctuations are negligibly small and in fact comparable to that in the unforced jet. These observations indicate that the minijet has reached a penetration depth of $y / D=-0.1$. With three adjacent minijets on $(N=3)$, the velocity fluctuations are appreciably larger in magnitude than their counterparts of $N=1$, and the maximum amplitude is shifted to a deeper position, i.e. from $y / D=-0.1$ at $N=1$ to $y / D=0$ at $N=3$. The fluctuations are now discernible at $y / D=0.2$, indicating an increased penetration depth, though the minijets clearly have not impinged on the wall opposite to the injecting minijets. With $N$ increasing to 4 , the maximum magnitude of the velocity fluctuations is appreciably larger than that of $N=3$, and again occurs at the centre $(y / D=0)$, where all minijets contribute to an increase in the velocity fluctuations. Furthermore, the fluctuations are now even discernible at $y / D=0.3$. It is worth pointing out that we did not move the hot wire closer to the wall because of its high fragility; therefore, we could not tell whether the minijets have penetrated through the main jet in this case. At $N=6$, the velocity fluctuations display symmetry about the centre, the maximum magnitude exceeding all other cases and taking place at the centre.

\subsection{Power spectral density function and minijet number}

Figure 5 compares $E_{u}$ measured on the centreline at $x / D=0.05$ with and without the main jet operated, where the $\log -\log$ scale is used to emphasize the low-frequency components. This function $E_{u}$ yields $\overline{u^{2}}=\int_{0}^{\infty} E_{u} \mathrm{~d} f$, where $f$ is frequency. In figure $5 e$, $E_{u}$ measured in the unforced jet shows a pronounced peak at $f_{0}$, indicating clearly the occurrence of the preferred mode structure. When the minijets as well as the main jet are operated, $E_{u}$ exhibits more pronounced peaks at $f / f_{a}=1$ and its harmonics. These observations result from the interaction between the main jet and minijet, referred to as the parametric resonance by Huang \& Hsiao (1999). Evidently, the unsteady injection produces the periodic structures upstream of the nozzle exit, as noted by Zhou et al. (2012). With increasing $N$, the peaks become more pronounced and occur at more harmonics, echoing the enhanced periodic structures (figure 4) and, hence, the enhanced excitation of the shear layer. The predominant frequencies do not vary with $N$ though. Note that $E_{u}$ is normalized by $\overline{u^{2}}$ so that its integration over the entire frequency range is always equal to unity. As a result, $E_{u}$ drops appreciably over the low frequency range.

\subsection{Fluctuating velocity and minijet number}

The number and configuration of minijets may profoundly affect the main jet issuing from the nozzle, as in the case of passive delta tabs (Zaman et al. 1994). This effect plays an important role in the downstream evolution of flow. As such, the radial profiles of the hot-wire measured root-mean-square (r.m.s.) velocity $u_{r m s}$ at $x / D=0.05$ are examined in the manipulated jets, along the $y$ and $z$ axes, respectively, for $N=1,2,3,6$. The data of the unforced jet are also presented for the purpose of comparison. Given the symmetrically arranged minijets $(N=2,6)$ about the $x-z$ plane (figure $6 b 1, d 1$ ), the $u_{r m s}$ distributions along the $y$-axis exhibit reasonable symmetry. The $u_{r m s}$ displays a pronounced peak at about $y / D= \pm 0.45$, where the shear layer is expected, in the injection or $x-y$ plane for $N=2$ (figure $6 b 1$ ), but remains unchanged in the orthogonal or $x-z$ plane (figure $6 b 2$ ), indicating that the shear layer between the two minijets is essentially undisturbed. Being symmetrical about the $z$-axis, the $u_{r m s}$ distributions are given only for $z / D \geqslant 0$ in figure 6(a2-d2). A broad bump 
(a)

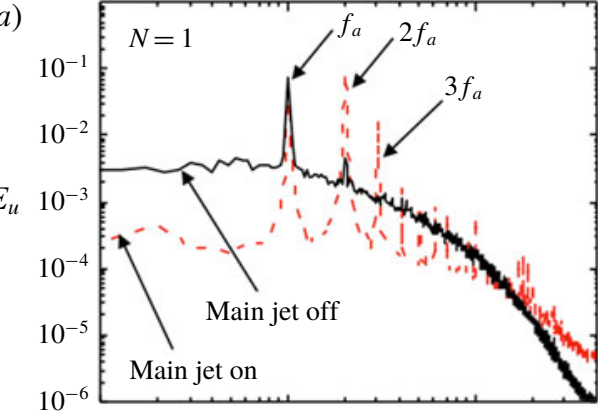

(c)

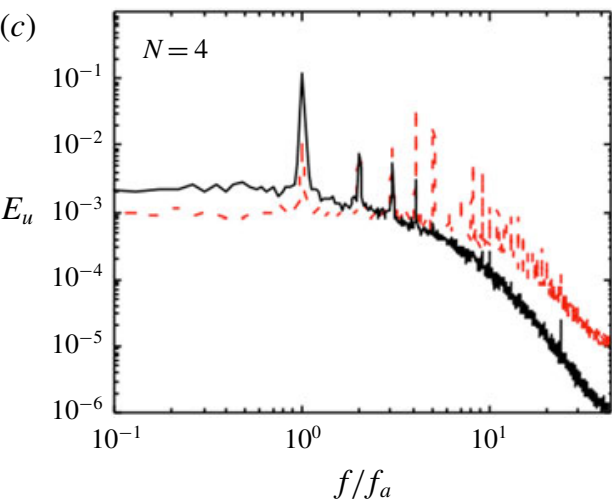

(b)

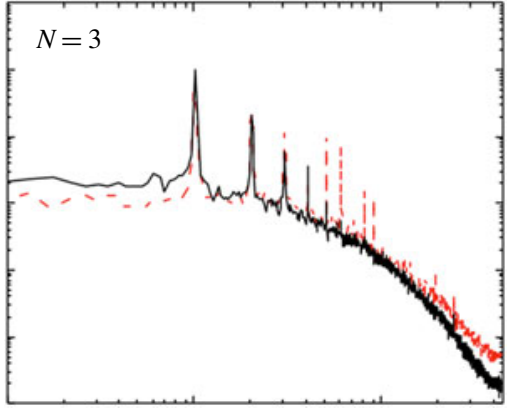

(d)

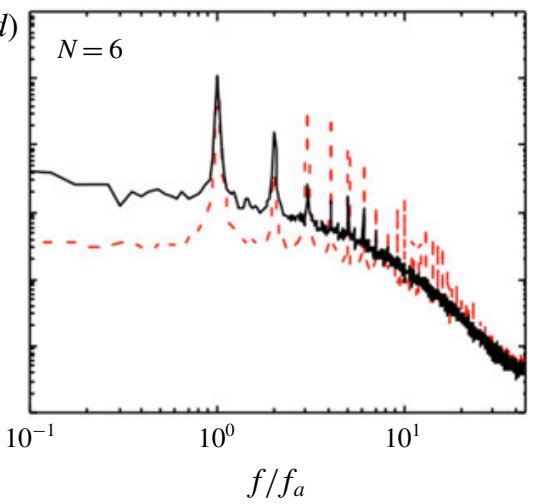

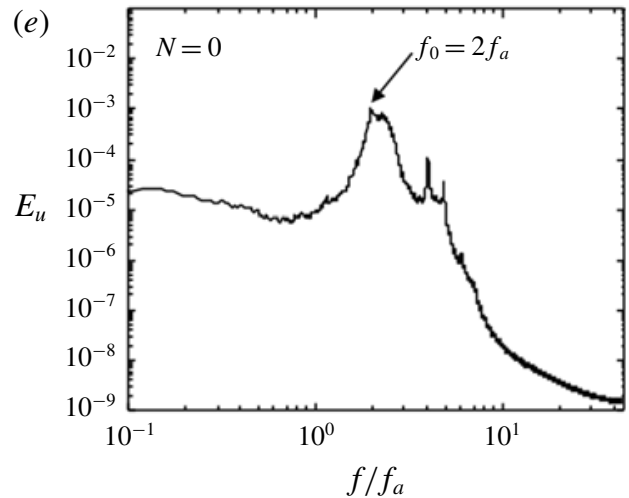

FIGURE 5. Power spectral density function $E_{u}$ of hot-wire signals $u$ measured at $(x / D, y / D, z / D)=(0.05,0,0)$ with and without the main jet: (a) $N=1$, (b) $N=3$, (c) $N=4,(d) N=6$, (e) $N=0,(x / D, y / D, z / D)=(3,0,0)$.

is evident at $y / D \approx 0.2$ for $N=2$ (figure $6 b 1$ ). The flow structure induced by an unsteady injecting minijet is similar to a pulsed jet in cross-flow, which forms a series of periodical vortex rings (M'closkey, King \& Cortelezzi 2002). It seems that these minijet-produced periodic vortices may occur most likely at $y / D \approx 0.2$, accounting for the broad bump. For $N=3$ and 6 , this bump moves to near the centre, with a significantly increased magnitude (figure $6 c 1, d 1$ ). Two factors may be responsible for this increase. Firstly, as the separation angle $\theta$ decreases from $180^{\circ}$ to $120^{\circ}$ and then $60^{\circ}$, two neighbouring minijets become close and their induced unsteady flows 

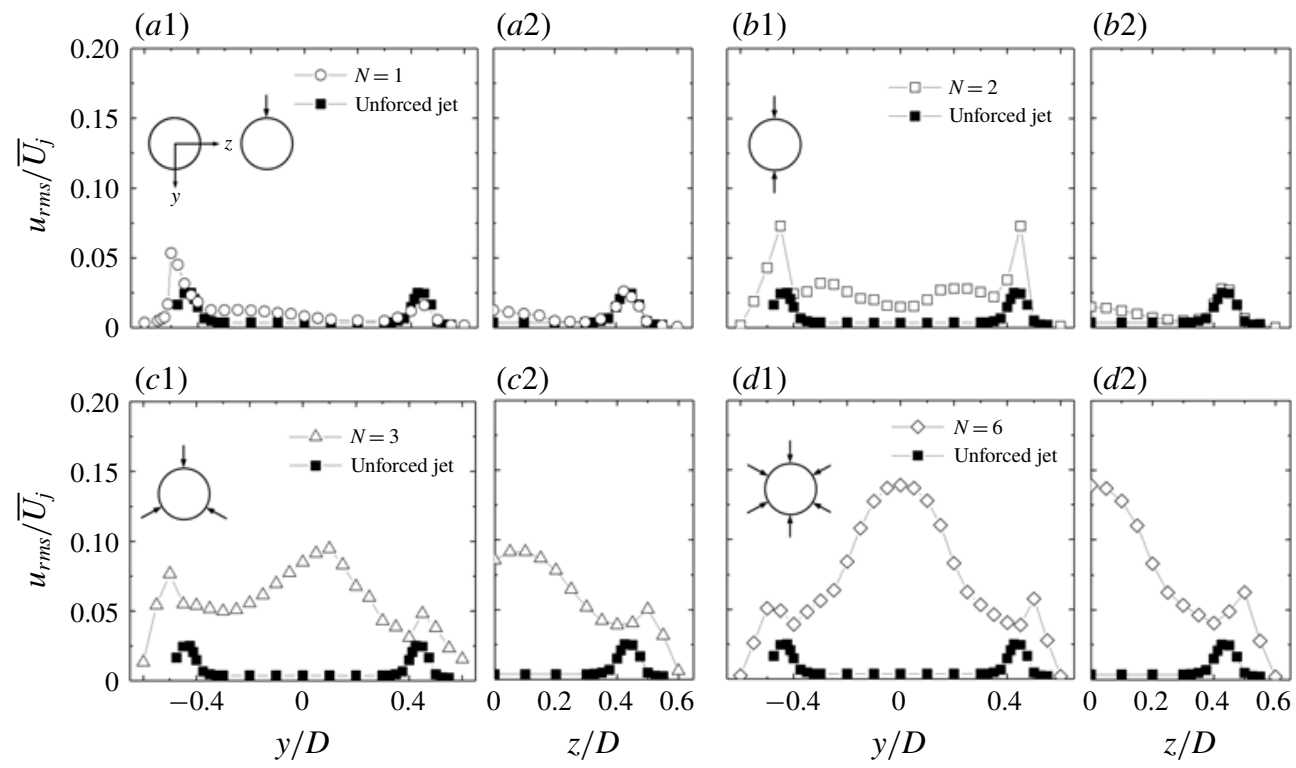

FIGURE 6. Radial distributions of fluctuating velocity $u_{r m s} / \overline{U_{j}}$ measured at $x / D=$ $0.05\left(f_{e} / f_{0}=0.5, C_{m}=1.2 \%\right)$ depend on minijet number: $(a 1-d 1)$ along the $y$-axis, $(a 2-d 2)$ along the $z$-axis.

interact more and more intensely. Zaman et al. (1994) noted that, as the neighbouring delta tabs approach each other, streamwise vortices interact more vigorously, resulting in the jet core fluid ejection. Secondly, as demonstrated in figure 4, every minijet generates a velocity fluctuation at the centre. For $N=6, \theta$ is smallest and all six minijets contribute to flow perturbations, thus producing the most pronounced bump at $y / D=0$.

\section{Artificial intelligence control system}

\subsection{Artificial intelligence control system}

Artificial intelligence methods allow us to explore the rich universe of nonlinear actuation mechanisms opened by independent spatially distributed actuators. Hence, we see the actuation and sensing hardware and control logic as intimately interwoven. The AI control system is sketched in figure 7. Generally, a control system facilitates a control goal for a plant by control hardware and a control logic/controller. The control hardware includes sensors and actuators as discussed in $\S 2$. This hardware monitors the plant output (velocity signals) and executes instructions from the controller. The open-loop arrangement is shown in figure 1(a) for calculating the cost value $J=\bar{U}_{5 D} / \bar{U}_{j}$. A minimized cost $J$ corresponds to the maximized decay rate $K=1-J$ of jet centreline mean velocity, which is an indicator of the mixing efficacy of a jet (Perumal \& Zhou 2018).

\subsection{Control optimization using linear genetic programming}

The six-dimensional vector $\boldsymbol{b}=\left[b_{1}, b_{2}, \ldots, b_{6}\right]^{\dagger}$ comprises all actuation commands. The $i$ th minijet is ' $\mathrm{ON}$ ' if the actuation command $b_{i}$ is positive and is 'OFF' otherwise. 


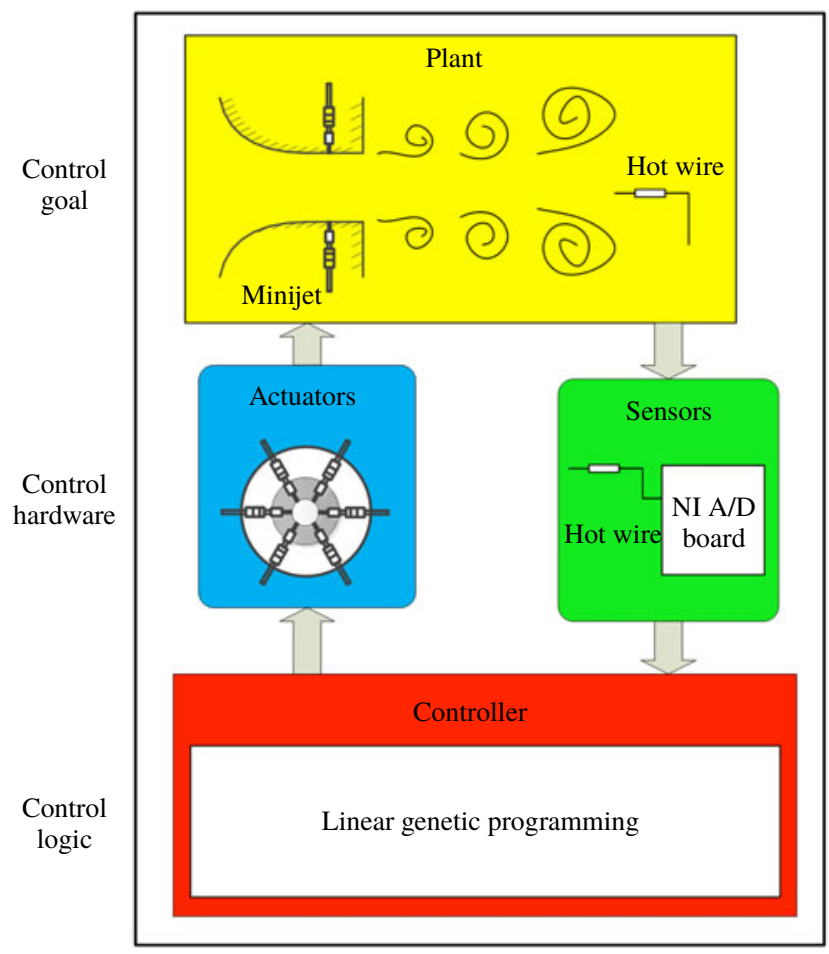

FIGURE 7. Principle sketch of the artificial intelligence control which consists of a plant (yellow), sensors (green), actuators (blue) and a controller (red) that includes a linear genetic programming (LGP) algorithm or other machine learning methods.

In the sequel, we assume that $b_{i}=1$ for ' $\mathrm{ON}$ ' and $b_{i}=0$ for 'OFF'. Following Wu et al. (2018a), we search for a control law including sensor feedback with hot-wire signals $\boldsymbol{s}$, multi-frequency open-loop forcing with harmonic functions contained in $\boldsymbol{h}=$ $\left[h_{1}, h_{2}, \ldots, h_{6}\right]^{\dagger}$. Here, $h_{i}=\sin \left(\omega_{a} t-\phi_{i}\right), i=1,2, \ldots, 6$, where $t$ is time, $\omega_{a}$ is a reference frequency to be determined in $\S 5.1$ and $\phi_{i}$ is the phase. Then,

$$
\boldsymbol{b}=\boldsymbol{K}(\boldsymbol{s}, \boldsymbol{h}),
$$

where the vector function $\boldsymbol{K}=\left[K_{1}, \ldots, K_{6}\right]$ comprises the actuation laws for each minijet. The time-averaged duty cycle of the $i$ th minijet is determined by the control law $\boldsymbol{K}$ and the arguments, i.e. sensor signals and harmonic functions. For open-loop forcing $\boldsymbol{b}=\boldsymbol{K}(\boldsymbol{h})$, the duty cycle of the $i$ th minijet becomes the sensor-independent time-average of the actuation command $\bar{K}_{i}(h)$. Thus, helical forcing may have a particularly simple representation, e.g. $b_{i}=h_{i}$. In general, only two harmonic functions, typically $\sin \omega_{a} t$ and $\cos \omega_{a} t$, are sufficient for harmonic functions with arbitrary phases. Following Paschereit, Wygnanski \& Fiedler (1995) and others, we add the cosine and sine components of $\omega_{a} / 2$ and $\omega_{a} / 4$, yielding a ten-dimensional vector $h=\left[h_{1}, h_{2}, \ldots, h_{10}\right]^{\dagger}$. The nonlinear function $\boldsymbol{K}$ can create arbitrary higher harmonics, arbitrary phase relationships between $\omega_{a}, \omega_{a} / 2, \omega_{a} / 4$ and higher harmonics, e.g. $1-2 h^{10}=\cos \left(10 \omega_{a} t\right)$, as well as arbitrary sum and difference frequencies. The control optimization searches for a law of form (4.1) that minimizes the cost,

$$
\boldsymbol{K}^{\star}=\underset{\boldsymbol{K}}{\arg \min } J[\boldsymbol{K}] .
$$


The regression problem implies a search for a mapping from multiple inputs to a multiple-output signal. Even in case of a linear function this implies the optimization of a large number of parameters. We employ the powerful linear genetic programming (LGP) as a regression solver and take the same parameters for the control law representation and for the genetic operations as Wu et al. (2018a). The first generation of LGP, $n=1$, contains $N_{i}=100$ random control laws, also called individuals. Each individual ' $i$ ' is experimentally tested for $5 \mathrm{~s}$ to yield the measured cost $J_{i}^{n}$, where the superscript ' $n$ ' denotes the generation number. Subsequent generations are produced from the previous ones with genetic operations (elitism, crossover, mutation and replication) and tested analogously. In the case of elitism, top-ranking individuals pass directly to the next generation. Replication copies a stochastically selected number of individuals into the next generation, which acts to preserve some well performing individuals. Crossover involves two selected individuals and then produces two individuals, with part of their elements exchanged. This operation tends to generate better individuals by exploitation. For the mutation operation, the instructions of a selected individual are randomly changed. Both crossover and mutation serve to explore potentially new and better minima of $J$. After the in situ performance measurements, the individuals are renumbered in order of performance, $J_{1}^{n} \leqslant J_{2}^{n} \leqslant \cdots \leqslant J_{N_{i}}^{n}$, where the subscript $i$ represents the individual index and $N_{i}$ and $n$ respectively denote the size and number of generations. We have noted in trial tests that all the winning individuals always involve every actuator. Therefore, when generating the 100 individuals of the first generation, we exclude the possibility of a permanently inactive actuator to accelerate the learning process, that is, as a plant-specific rule, we discard and replace any individual for testing if one or more actuators are not active.

It is worth mentioning that the present jet control is formulated as a model-free regression problem: determine the law which minimizes the given cost function. The considered search space of control laws significantly extends hitherto considered actuations. Firstly, general multiple-input actuation is allowed without any imposed symmetry constraints. Thus, actuations with arbitrary combinations of minijets thereof can be realized. Secondly, the search space includes broadband multi-frequency actuation. Thirdly, nonlinear sensor feedback is included, which is made by nonlinear operations with the sensor signal $\boldsymbol{s}$, e.g. $\boldsymbol{b}=\log _{10}(\boldsymbol{s})$ (Wu et al. 2018a). However, this feature is not found improving appreciably the control performance and is therefore removed eventually in the learning process. Fourthly, the control law may include nonlinear combinations of multi-frequency forcing and sensor feedback. The key enabler for the control optimization in this search space is genetic programming as a powerful regression solver. Genetic programming may be considered as an example for the many powerful regression solvers of AI.

\subsection{Parameters and control landscape}

The LGP parameters for this study are displayed in table 1. These values are adopted from a previous MLC jet mixing study in the same facility with a single minijet (Wu et al. 2018a). The parameters are identical or close to the ones employed in numerous experimental studies as summarized by Duriez et al. (2016) and Noack (2019). Elitism is set to $N_{e}=1$, i.e. the best individual of a generation is copied to the next one. The replication, crossover and mutation probabilities are $10 \%, 70 \%$ and $20 \%$, respectively. The individuals on which these genetic operations are performed come from a tournament selection of size $N_{t}=7$. The instruction number varies from 10 to 50 . The operations comprise $+,-, \times, \div, \sin , \cos , \tanh , \log _{10}$ and $g^{2}$, where $g$ is 


Parameters
Individuals
Tournament size
Elitism
Crossover
Mutation
Replication
Min. instruction number
Max. instruction number
Operations
Number of constants
Constant range

Value
$N_{i}=100$
$N_{t}=7$
$N_{e}=1$
$P_{c}=70 \%$
$P_{m}=20 \%$
$P_{r}=10 \%$
10
50
$N_{i}=+,-, \times, \sin , \cos , \tanh , \log , g^{2}$
$N_{c}=3$
$[-1,1]$

Value

$N_{i}=100$

$N_{t}=7$

$P_{c}=70 \%$

$P_{m}=20 \%$

10

$[-1,1]$

TABLE 1. Linear genetic programming parameters employed for experiments. The symbol $g$ indicates an input argument.

the input argument. The operations $\div$ and $\log _{10}$ are protected to prevent an undefined expression with a vanishing argument; for example, $\log _{10}(g)$ is modified to $\log _{10}(|g|)$. In addition, LGP uses three random constants in the range $[-1,1]$.

The evolution of control laws is depicted with a proximity map following Duriez et al. (2016). The main idea is that the considered ensemble of $\boldsymbol{K}_{i}(\boldsymbol{h})$ is represented as points in a two-dimensional feature plane $\boldsymbol{\gamma}_{i}=\left(\gamma_{i, 1}, \gamma_{i, 2}\right)$, where $i=1,2, \ldots, N_{i} \times n$, so that the difference between the control laws is optimally indicated by the distance between feature vectors. The key is the definition of a metric $D_{i j}$ between the control laws $\boldsymbol{K}_{i}(\boldsymbol{h})$ and $\boldsymbol{K}_{j}(\boldsymbol{h})$. For the considered open-loop actuation, this metric is the rootmean-square averaged Euclidean difference between the actuation command vectors accounting for a potential time-delay, given by

$$
M_{i j}=\min _{\tau \in\left[0, T_{a}\right]} \sqrt{\left\|\boldsymbol{K}_{i}(\boldsymbol{h}(t))-\boldsymbol{K}_{j}(\boldsymbol{h}(t-\tau))\right\|^{2}} .
$$

In the employed metric, we incorporate also the control performance $J_{i}$ by a penalization term, i.e.

$$
D_{i j}=M_{i j}+\beta\left|J_{i}-J_{j}\right| \text {. }
$$

The parameter $\beta$ is chosen so that the maximum actuation distance $M_{i j}$ is equal to the maximum difference in the performance terms:

$$
\max _{i, j=1,2 \ldots N_{i} \times n} M_{i j}=\beta \max _{i, j=1,2 \ldots N_{i} \times n}\left|J_{i}-J_{j}\right| .
$$

Given the resulting configuration matrix $\boldsymbol{D}=\left(D_{i j}\right)\left(i, j=1,2, \ldots, N_{i} \times n\right)$, classical multi-dimensional scaling (Cox \& Cox 2000) uniquely determines feature vectors $\boldsymbol{\gamma}_{i}$, $i=1,2, \ldots, N_{i} \times n$, so that the distances are optimally preserved:

$$
\sum_{i=1}^{N_{i} \times n} \sum_{j=1}^{N_{i} \times n}\left(\left\|\boldsymbol{\gamma}_{i}-\boldsymbol{\gamma}_{j}\right\|-D_{i j}\right)^{2}=\min .
$$

The translational degree of freedom is removed by centring the feature vectors $\sum_{i=1}^{N_{i} \times n} \gamma_{i}=0$. The feature vectors are sorted and rotated so that the first coordinate 


$\begin{array}{lcc}\text { Mode } & \text { Benchmark forcing } & \text { AI control } \\ \text { Unforced jet } & J_{u}=0.974 & - \\ \text { Axisymmetric forcing } & J_{a}=0.665 & J_{1}^{1}=0.626 \\ \text { Helical forcing } & J_{h}=0.568 & J_{1}^{2}=0.555 \\ \text { Flapping forcing } & J_{f}=0.423 & J_{1}^{5}=0.419 \\ \text { Combined forcing } & - & J_{1}^{11}=0.305\end{array}$

TABLE 2. Cost function $J$ for different actuations at $R e_{D}=8000$.

has the largest variance, the second coordinate the second largest, etc. The coordinates are indeterminate by a sign (mirroring), like POD modes and their amplitudes.

Finally, a control landscape $J(\boldsymbol{\gamma})$ is interpolated from the three-dimensional data points $\left(\gamma_{i, 1}, \gamma_{i, 2}, J_{i}\right), i=1,2, \ldots, N_{i} \times n$. The two-dimensional feature vectors $\gamma_{i}$ are connected by an unstructured grid from a Delaunay (1934) triangulation. This triangulation guarantees that the mesh triangles are optimally equilateral. The $J$-values in each mesh triangle $i_{1}, i_{2}, i_{3} \in\left\{1,2, \ldots, N_{i} \times n\right\}$ are interpolated from the known values at the vertices $J_{i_{1}}, J_{i_{2}}, J_{i_{3}}$. These control landscapes have been employed in several AI-based control schemes (Kaiser et al. 2017). They indicate the complexity of the actuation response and the learning progress of AI-based control. Often, the feature coordinates can be linked with the physical properties of actuation a posteriori, thus providing additional insights.

\section{Outcome of the AI control}

\subsection{Representative reference actuations}

A few well-known reference forcings are firstly presented to facilitate the understanding of the AI learning process and highlight the uniqueness of this method. In our earlier studies, turbulent jet mixing has been optimized for the same cost function and experimental conditions. For single unsteady minijet forcing, the optimal $f_{a}$ is found to be $67 \mathrm{~Hz}$ (Wu et al. 2018a), 0.5f , and the optimal $C_{m}$ is $1.2 \%$ based on a dual-input-and-one-output closed-loop control technique (Wu, Wong \& Zhou 2018b). As such, we choose the same $f_{a}$ or $\omega_{a}=2 \pi f_{a}$ and $C_{m}=1.2 \%$ for every minijet. With $C_{m}$ fixed for each minijet, the overall mass flow of injected fluid in one actuation period $T_{a}$ is the same for all actuations, that is, the input/actuation energy is the same, irrespective of control modes or laws. Consider three reference forcings (e.g. Hilgers \& Boersma 2001; Yang \& Zhou 2016; Yang 2017), viz.

$$
\begin{gathered}
\text { axisymmetric forcing } b_{i}=h_{1}-\alpha_{a}, \quad i=1,2, \ldots, 6 ; \\
\text { helical forcing } b_{i}=h_{i}-\alpha_{h}, \quad i=1,2, \ldots, 6 ; \\
\text { flapping forcing } b_{1}=b_{2}=b_{3}=h_{1}-\alpha_{f}, \quad b_{4}=b_{5}=b_{6}=h_{4}-\alpha_{f} \text {. }
\end{gathered}
$$

The constants $\alpha_{a}, \alpha_{h}$ and $\alpha_{f}$ correspond to the duty cycles and have been optimized with respect to the cost. As mentioned before, actuation is performed only when $b_{i}>0$. The cost functions are found to be $J_{a}=0.665, J_{h}=0.568$ and $J_{f}=0.423$ for the optimized axisymmetric, helical and flapping forcings (table 2), respectively, based on the conventional open-loop control, which provide the benchmarks for the AI control performance to be discussed below. 


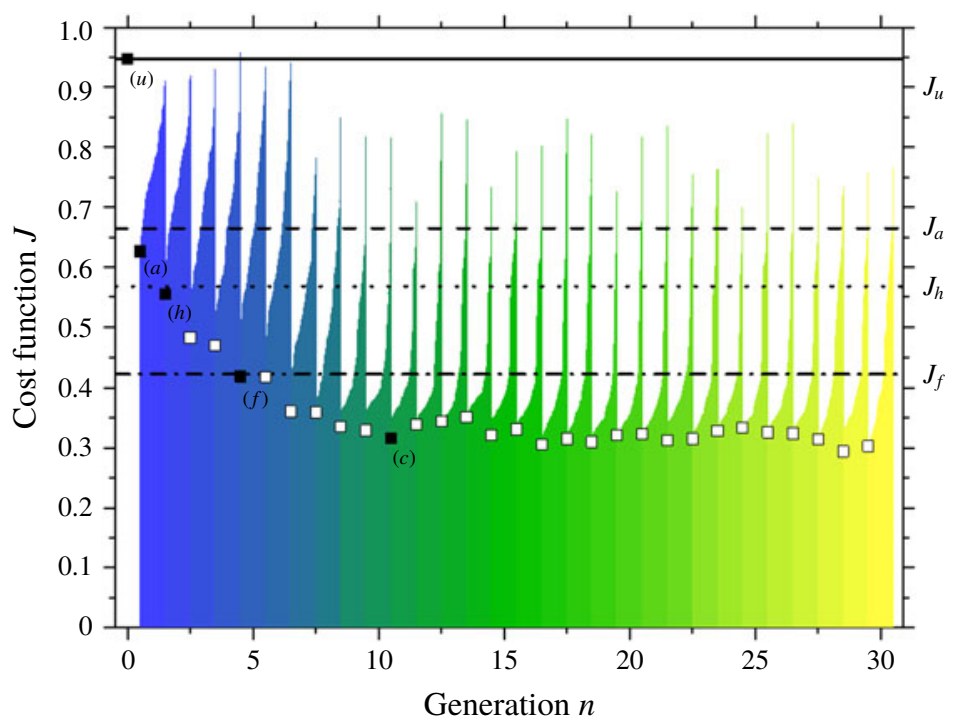

FIGURE 8. Learning curve (3000 individuals) of AI control for $(u)$ unforced jet, (a) axisymmetric forcing, $(h)$ helical forcing, $(f)$ flapping forcing and $(c)$ combined forcings. Here $J_{u}, J_{a}, J_{h}$ and $J_{f}$ are costs corresponding to the benchmarks of unforced, open-loop axisymmetric, helical and flapping forcings, respectively (see table 2).

\subsection{Learning process of AI control}

In the initial stage of the learning process we included a feedback signal $s(t)=$ $\left[u_{3 D}, u_{5 D}\right]$ as one input (equation (4.1)) for the AI system, where $u_{3 D}$ and $u_{5 D}$ are the fluctuating velocity signals measured at $x / D=3$ and 5 , respectively. It is found from dozens of experiments that the search for the optimal solution benefits neither from the subharmonic components of $h_{7}, \ldots, h_{10}$ nor from the feedback signals. Therefore, the AI control laws (4.1) may be cast in the periodic open-loop form, $b_{i}=$ $K_{i}\left(h_{1}, h_{2}, \ldots, h_{6}\right), i=1,2, \ldots, 6$. Hence, we restrict our following discussion to this open-loop actuation.

The learning curve of the AI control is presented in figure 8 , where the square symbol marks the first and best individual of each generation with $N_{i}=100$ control laws. The remaining costs grow monotonously with their indices, and the 100 individuals of each generation form a colour bar. The square symbol curve unveils the best performance from generation $n=1$ to 30 . The best individual of the first generation or stage 1 is characterized by an axisymmetric control law (see (A 1) in appendix A).

This law is equivalent to $(5.1 a)$ except for a time shift, reflected by $4 / 6 \pi$. The performance $J_{1}^{1}=0.626$ (see figure 8 and table 2) is slightly better, about $5.8 \%$ lower, than the benchmark of axisymmetric forcing (5.1a), though much higher than that $\left(J_{u}=0.947\right.$ or $\left.K \approx 0.05\right)$ of the unforced jet. Note that the centreline mean velocity for calculating $K$ or $J_{a}$ is measured over a duration of $60 \mathrm{~s}$ in the benchmark experiments, but only $5 \mathrm{~s}$ for estimating $J_{i}$ as the measured $J_{i}$ is used to evaluate control laws and does not need to be very accurate in the learning process of AI control. An accurate long-time evaluation of $J$ is performed only in the last generation $n=30$. This difference in evaluating the cost function could account for the deviation between $J_{1}^{1}$ and $J_{a}$. However, the AI control or specifically genetic programming breeds several 
copies of the winning individual, covering all possible combinations of the control parameters, and then takes the best performing one. This process differs in essence from the searching process of the conventional open-loop control which optimizes one control parameter first and then moves to next with the first parameter fixed. The advantage of the former over the latter is obvious. Therefore, different search strategies cannot be excluded from the mechanisms behind the deviation, which will be further substantiated by the fact that all the best cost functions of the different stages in the learning curve are less, albeit slightly, than their corresponding benchmarks produced from the conventional open-loop control.

Stage 2 starts with the second generation when the AI control discovers a better performing helical forcing (equation (A 2) in appendix A). This forcing differs in form from $(5.1 b)$, but clearly shows a uniformly travelling wave in the azimuthal direction (to be demonstrated in $\S 5.3$ ), its cost $J_{1}^{2}$ being again slightly lower than $J_{h}$ (table 2). Helical forcing reduces $J$ further as found from the numerical simulation study of a similar jet mixing optimization (Hilgers \& Boersma 2001). Local spatial stability analysis indicates that, unlike axisymmetric forcing, helical perturbations are spatially amplified downstream of the potential core (Garnaud et al. 2013).

Flapping forcing takes place in stage 3, starting from the fifth generation. The law (equation (A 3) in appendix A) is similar to (5.1c) but incorporates an asymmetry. An optimized asymmetry yields a reproducibly better mixing, again $J_{1}^{5} \leqslant J_{f}$ (table 2 ).

The eleventh generation marks the emergence of stage 4. The AI control discovers a very sophisticated control law. See (A 3) in appendix A. This forcing significantly outperforms the flapping forcing found in generation 5, the corresponding $J_{1}^{11}$ plunging to 0.305 , a drop of $27 \%$ compared with the smallest $J_{1}^{5}$ in generation 5 and less than $1 / 3$ of the unforced jet. The actuation mechanism does not change any more in following generations with little variation in costs, pointing to the convergence of the AI learning process. It is worth highlighting that this actuation mechanism is reproducible, that is, approximately the same converged cost has been observed in all experiments, notwithstanding a change in the initial parameters of the first generation. However, not all AI learning curves go through the stages of axisymmetric, helical and flapping forcings; some AI experiments may find only two of the three stages in the learning process.

\subsection{Representative control laws and flow responses}

The control mechanisms may be elucidated from the analysis of the spatio-temporal actuations, shown in figure 9, extracted from the control laws of $n=1,2,5$ and 11 . Each circular pie corresponds to one sixth of the excitation period, while its six sectors represent the six minijets. The arrow indicates that the minijet is on and the radial depth of the blue area is proportional to the duration when the minijet is injecting. The spatio-temporal actuation is found to be internally consistent with the cross-sectional and streamwise flow structure shown in figures 10 and 11. Axisymmetric forcing (figure 9a1-a6) is characterized by simultaneous blowing of all minijets and a small $\alpha$ of $13.3 \%$. As a result, the cross-sectional flow structure (figure 10a1-a6) is axisymmetric, and the ring vortex is evident. Yang \& Zhou (2016) discussed in detail the distortion, formation of longitudinal structures and generation of mushroom-like structures in the braid region between ring vortices, and presented a scenario on how the interactions between the longitudinal structures, mushroom-like structures and ring vortices enhance entrainment and mixing. The six synchronized minijet excitations greatly strengthen the ring vortices, as shown in figure 11(a) (cf. figure $11 u$ ). 


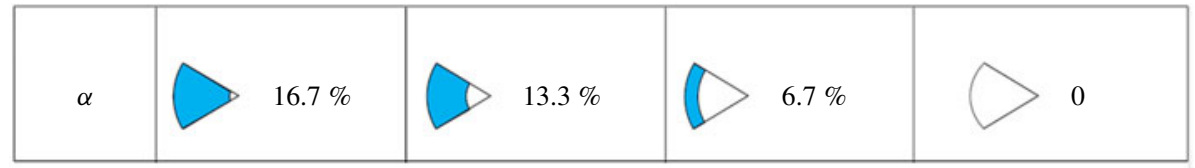

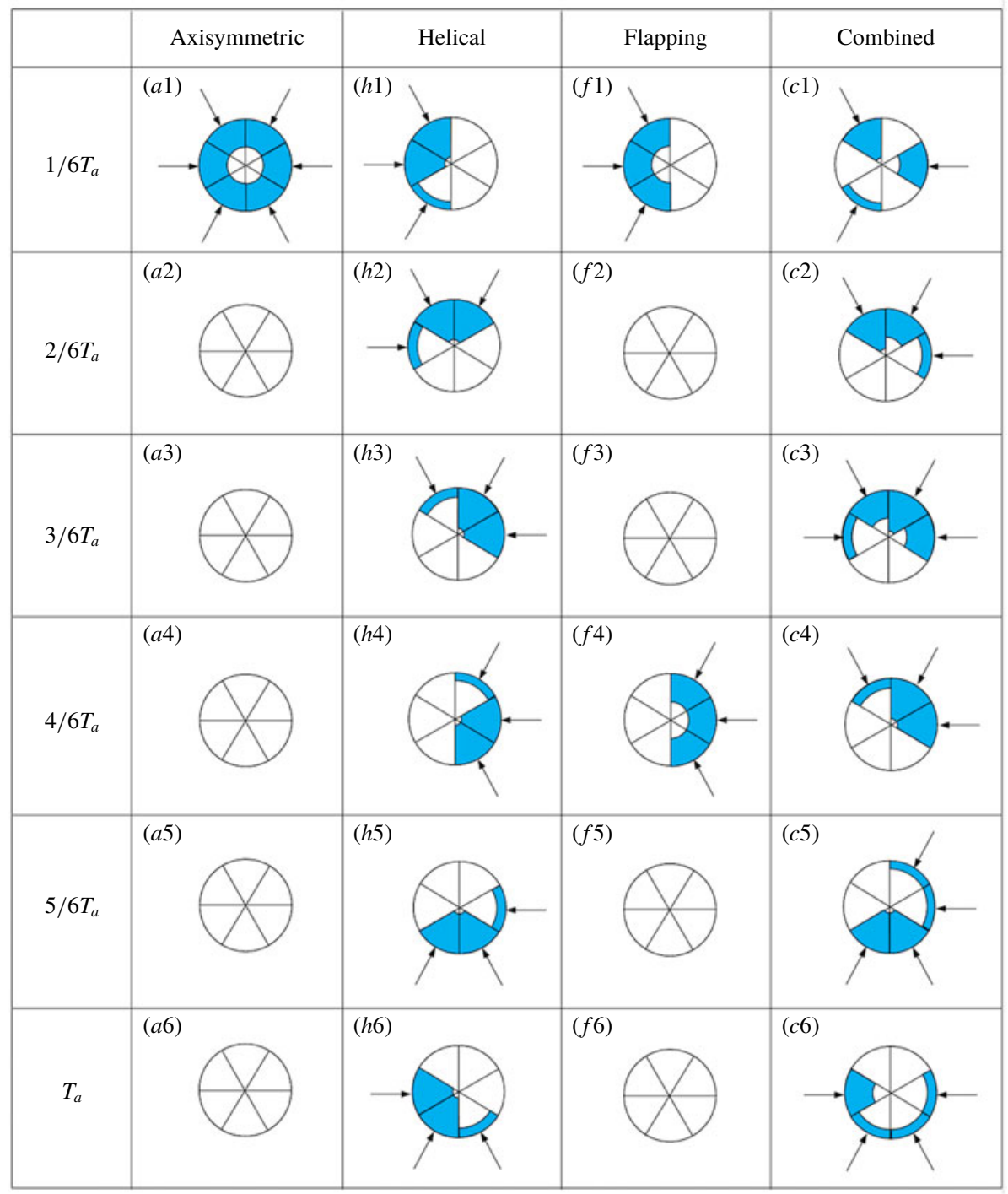

FIGURE 9. Actuation associated with the best individuals of generation $n=1,2,5$ and 11 of figure 8. From top to bottom: six instances associated with $\phi=$ $T_{a} / 6,2 T_{a} / 6,3 T_{a} / 6, \ldots, T_{a}$ in one excitation period. Each of the six sectors of the circles corresponds to one minijet.

For helical forcing (figure $9 h 1-h 6$ ), two or three minijets are blowing simultaneously at any instant, with $\alpha$ reaching $40 \%$. These blowing actions rotate clockwise from (h1) to (h6). The greatly increased $\alpha$, probably required for the generation of helical motion, may act to inhibit the occurrence of mushroom-like structures (Perumal \& Zhou 2018), which are absent in figure $10(h 1-h 6)$. The jet (figure $11 h)$ exhibits 


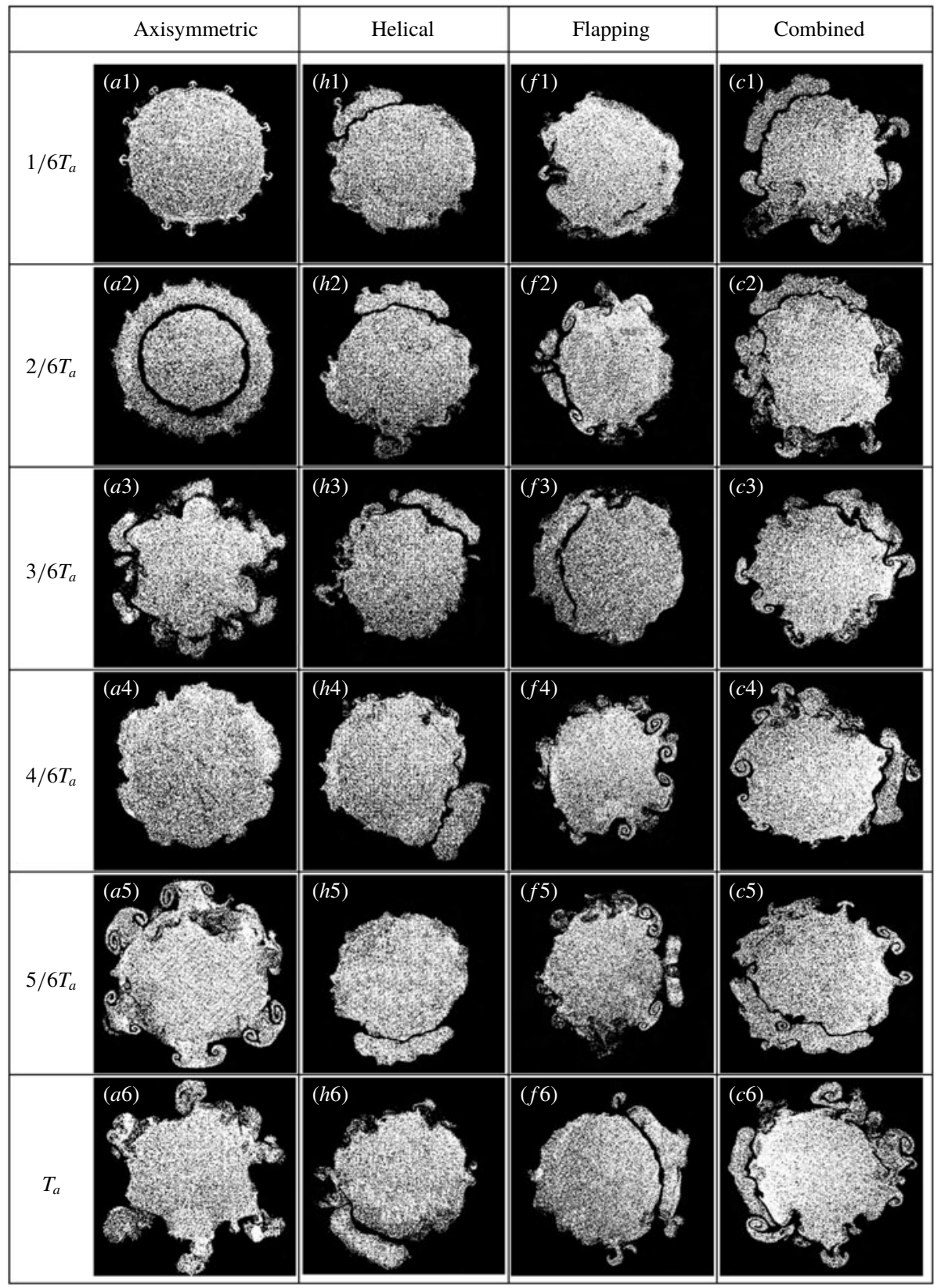

FIGURE 10. Sequential photographs of the cross-sectional flow structure captured at $x^{\star}=$ 0.25 . From top to bottom: six instances at $t_{i}=i T_{a} / 6(i=1,2, \ldots, 6)$ in one actuation period $T_{a}\left(=1 / f_{a}\right)$.

more spread than axisymmetric forcing (figure 11a). In case of flapping forcing (figure $9 f 1-f 6$ ), three adjacent minijets are blowing simultaneously at one instant with $\alpha=13.3 \%$ and are switched to the other three after a phase shift of $\pi$, thus creating the asymmetric flapping jet column (figures $10 f 1-f 6,11 f 1-f 2$ ). The actuation configuration of the rightmost column $(n=11)$ in figure 9 is complex. Firstly, the 
(u) Unforced jet

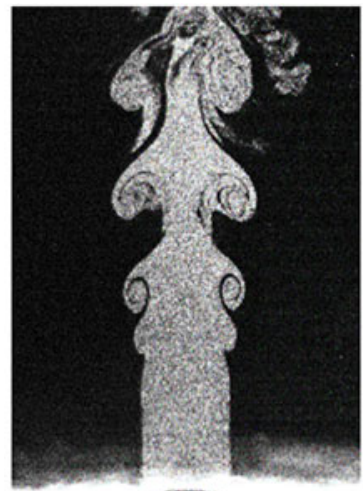

(f1) Flapping plane

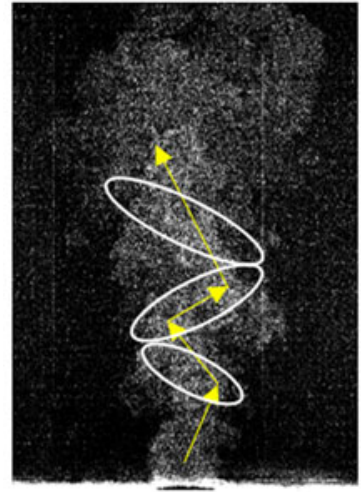

(a) Axisymmetric

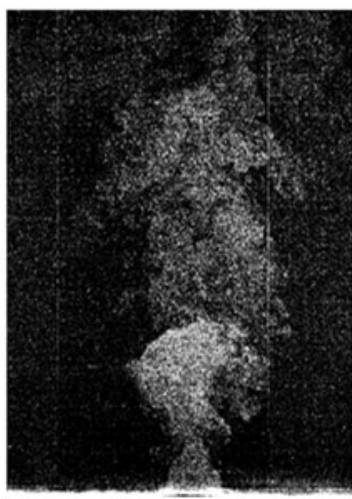

( $f 2$ ) Flapping plane
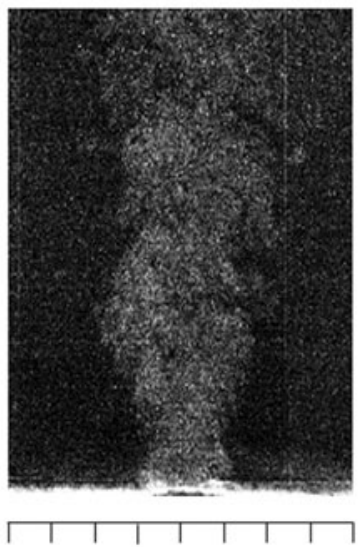

(h) Helical

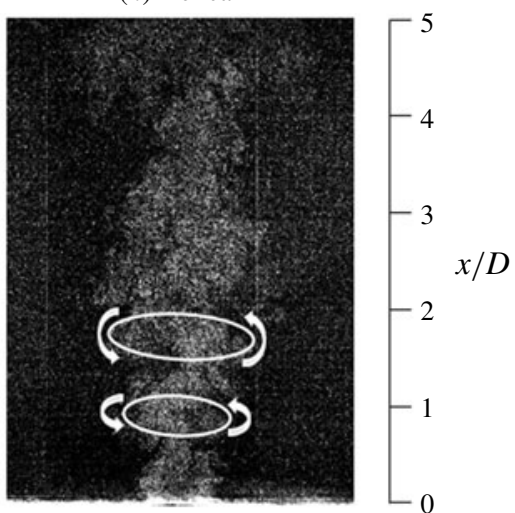

(c) Combined
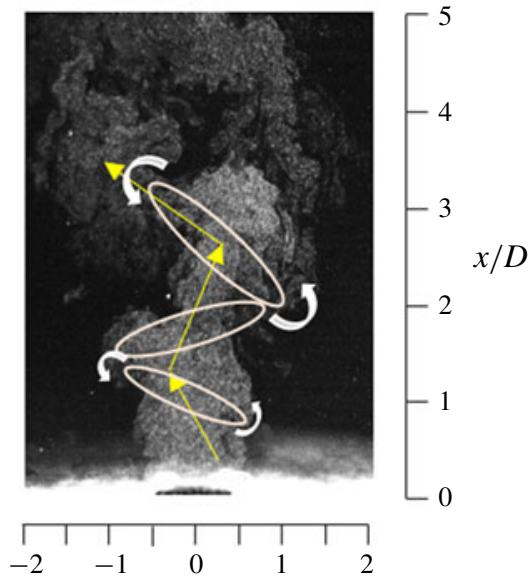

$y / D$ or $z / D$

FIGURE 11. Flow visualization of $(u)$ unforced jet, $(a)$ axisymmetric, $(h)$ helical, $(f)$ flapping and $(c)$ combined forcings from figure 8, respectively. White ellipses and crooked arrows indicate vortex rings and their rotation, respectively, and yellow arrows highlight the jet column oscillation.

number of simultaneously injecting minijets can be $1,2,3$ or 4 , mostly adjacent to each other. Secondly, the injecting minijets tend to rotate clockwise. The resulting effect is to produce both helical and flapping motions (figures 10c1-c6, 11c). For convenience, hereinafter we refer to this forcing as the combined mode. Thirdly, $\alpha$ varies from one blowing minijet to another, from about $6.7 \%$ to $53 \%$. The effect could be twofold. On one hand, the varying duty cycles of the blowing minijets may yield a resultant blowing force not going through the jet centreline (figure $9 c 1-c 6$ ). This may produce a precession effect, causing additional jet column oscillation (Wong, Nathan \& O'Doherty 2004). On the other hand, whilst a small $\alpha$ facilitates the generation of mushroom-like structures (figure 10c1-c6), a large value enhances the strength of the flapping motion (figure $11 \mathrm{c}$ ), as noted by Perumal \& Zhou (2018). All the features, confirmed by more detailed analysis in $\S 6$, act to promote mixing, consistent with the observed minimum $J$ (figure 8). The generation of such a sophisticated control mode, along with the generation of a complex turbulent flow 


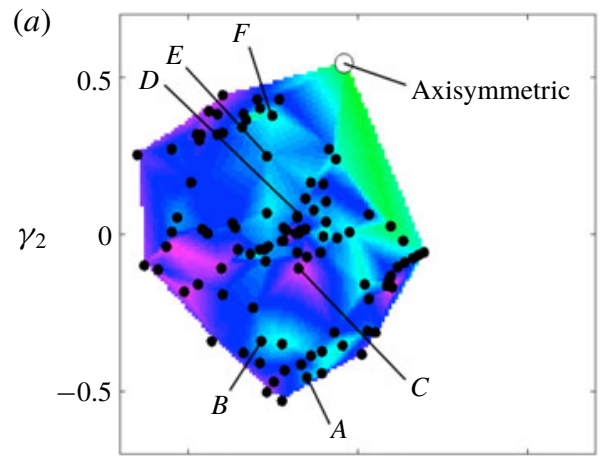

(b)

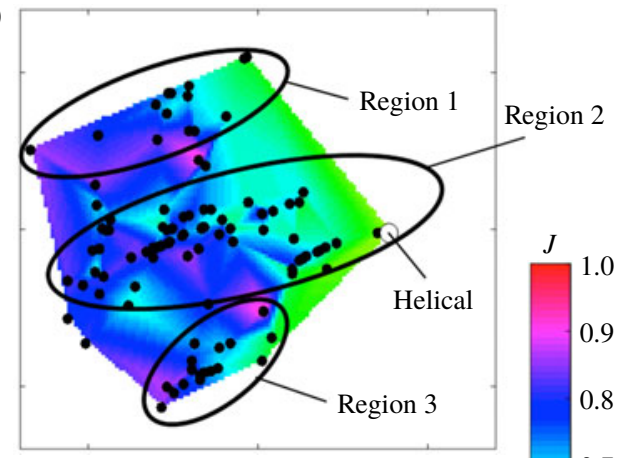

(d)

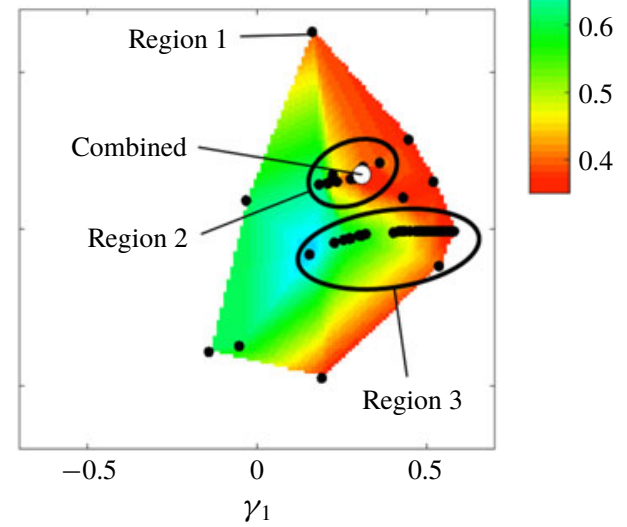

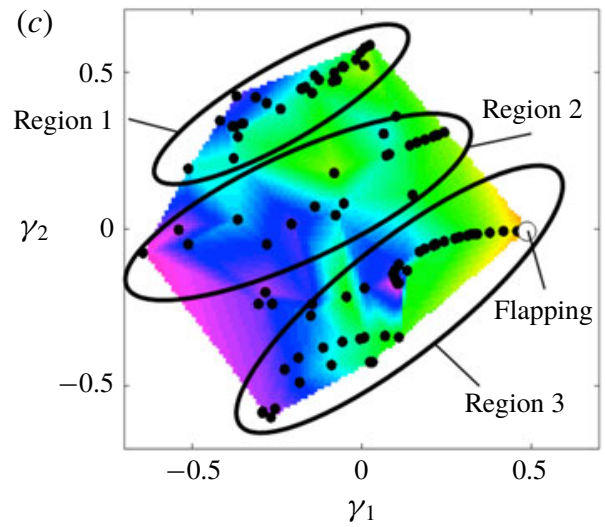

FIGURE 12. Control landscape associated with generations 1, 2, 5 and 11 (400 individuals). Each symbol represents an individual control law. The colour scheme corresponds to the cost value $J$ of the control laws, e.g. (a) $n=1$, (b) $n=2$, (c) $n=5$, (d) $n=11$. The white circle of bigger size corresponds to the best individual of one generation. The elliptic contours enclose similar control laws.

structure, would have been extremely challenging for conventional control techniques, be it open- or closed-loop and model-based or model-free approaches.

\subsection{Control landscape: cartographing all actuation laws}

Proximity maps $(\$ 4.3)$ provide a very revealing illustration of the evolution process of the control laws. The underlying metric between two control laws $\boldsymbol{b}$ and $\boldsymbol{b}^{\prime}$ is given by $D_{i j}$ (4.4). Figure 12 presents the proximity map of the control laws in a twodimensional plane such that this metric is optimally preserved. This plane is spanned by the feature coordinates $\gamma_{1}$ and $\gamma_{2}(\S 4.3)$, which are derived from a mathematical optimization process. The details of deriving $\gamma_{1}$ and $\gamma_{2}$ from individual generations are given in Cox \& Cox (2000). Physically, the distance between two points, which are given in terms of $\left(\gamma_{1}, \gamma_{2}\right)$, in the plane is directly linked to the extent of how closely similar to each other two control laws are.

The subfigures display the feature coordinates of the four discussed generations ( $n=1,2,5$ and 11). Interesting observations and inferences could be made from the subfigures. For $n=1$ (figure $12 a$ ), the points appear rather randomly distributed. The optimal solution of axisymmetric forcing, indicated by an open circle, occurs at 
$\left(\gamma_{1}, \gamma_{2}\right)=(-0.04,0.54)$, where $\gamma_{2}$ is the largest of all points. At $n=2$ (figure $\left.12 b\right)$, these points appear forming three separated regions numbered 1 through 3 , as enclosed by the elliptic contours, where most of the control laws fall in. The optimal solution that appears in region 2 and corresponds to helical forcing now occurs at $\left(\gamma_{1}, \gamma_{2}\right)=(0.39,-0.01)$, where $\gamma_{1}$ is the largest of all points. At $n=5$, the individual points tend to populate along discrete curves (figure 12c), which is a commonly observed phenomenon of the AI control, e.g. Li et al. (2017) and Wu et al. (2018a). The optimal solution now takes place at $\left(\gamma_{1}, \gamma_{2}\right)=(0.49,-0.01)$, where $\gamma_{1}$ is again the largest of all, within region 3, and corresponds to flapping forcing. It has been confirmed that most of the data points within regions 1,2 and 3 correspond to axisymmetric, helical, and flapping forcings, respectively. By $n=11$ (figure $12 d$ ), the optimal solution occurs at $\left(\gamma_{1}, \gamma_{2}\right)=(0.31,0.17)$ within region 2 , corresponding to the combined mode. It is noteworthy that those points in this region now correspond to either helical or combined forcing; this very fact may suggest that the so-called combined forcing may have been developed from and more closely related to helical forcing. Interestingly, region 1 , where axisymmetric forcing takes place, contracts to a single point, while region 2 or 3 is reduced to a curve.

The cost $J$ associated with each point is colour coded from red $(J=0)$ to pink $(J=1)$, as indicated by the colour bar. The cost values within each coloured area are interpolated from the 100 individuals in each generation. The control landscape at $n=1$ displays quite a few minima, as indicated by the green coloured areas, in the feature plane (figure 12a), suggesting the complexity of the learning task in the early stage, internally consistent with the rather random distribution of the points. The landscape becomes simpler towards the rightmost boundary of the generation. As $n$ increases, the individuals tend to line up on the ridge-curves marking the cost valleys.

The feature coordinates have technically no a priori meaning. However, an approximate physical meaning of $\gamma_{1}$ and $\gamma_{2}$ may be inferred from the careful analysis of the control laws. The coordinate $\gamma_{1}$ is correlated with the degree of asymmetric forcing, while $\gamma_{2}$ is linked to the number of simultaneously injecting minijets. Consider six arbitrarily chosen control laws $A-F$ in the first generation (figure 12a). Figure 13 shows the on- and off-states of the minijets corresponding to the six control laws. Evidently, the maximum number of simultaneously injecting minijets increases from one (law $A$ ) to six (law $F$ ).

\section{Discussion: representative control laws and flow structures}

\subsection{Jet spread and predominant flow structures}

Axisymmetric forcing at $n=1$ (figure 11a) leads to an early disruption of the potential core and a significantly improved entrainment, as suggested by the substantially reduced 'neck' due to ambient fluid (dark colour) brought into the jet by the greatly increased strength of vortices near the nozzle exit. The flow structure exhibits an appreciably increased lateral spread than the unforced jet (figure $11 u$ ). The $E_{u}$ of the centreline $u$ (figure $14 a$ ) measured at $x / D=1.0$ displays the most pronounced peaks of all at $f / f_{a}=1.0$ and its harmonics, though the peaks at higher harmonics vanish rapidly from $x / D=1.0$ to 4.0 . These peaks become very sharp because of the minijet excitation. The ring vortices remain axisymmetric (figure 10a1-a6), which is corroborated by the spectral phase $\Phi_{12}$, about zero over the entire range of $f / f_{a}=0-2.0$, between two simultaneously measured hot-wire signals $u_{1}$ and $u_{2}$ at $x / D=1$ and $y / D= \pm 0.3$ (figure $15 a$ ). The $\Phi_{12}$ is calculated by $\tan ^{-1}\left(Q_{12} / C o_{12}\right)$, 


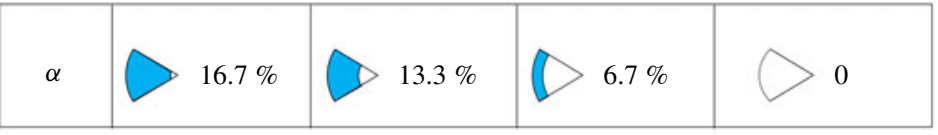

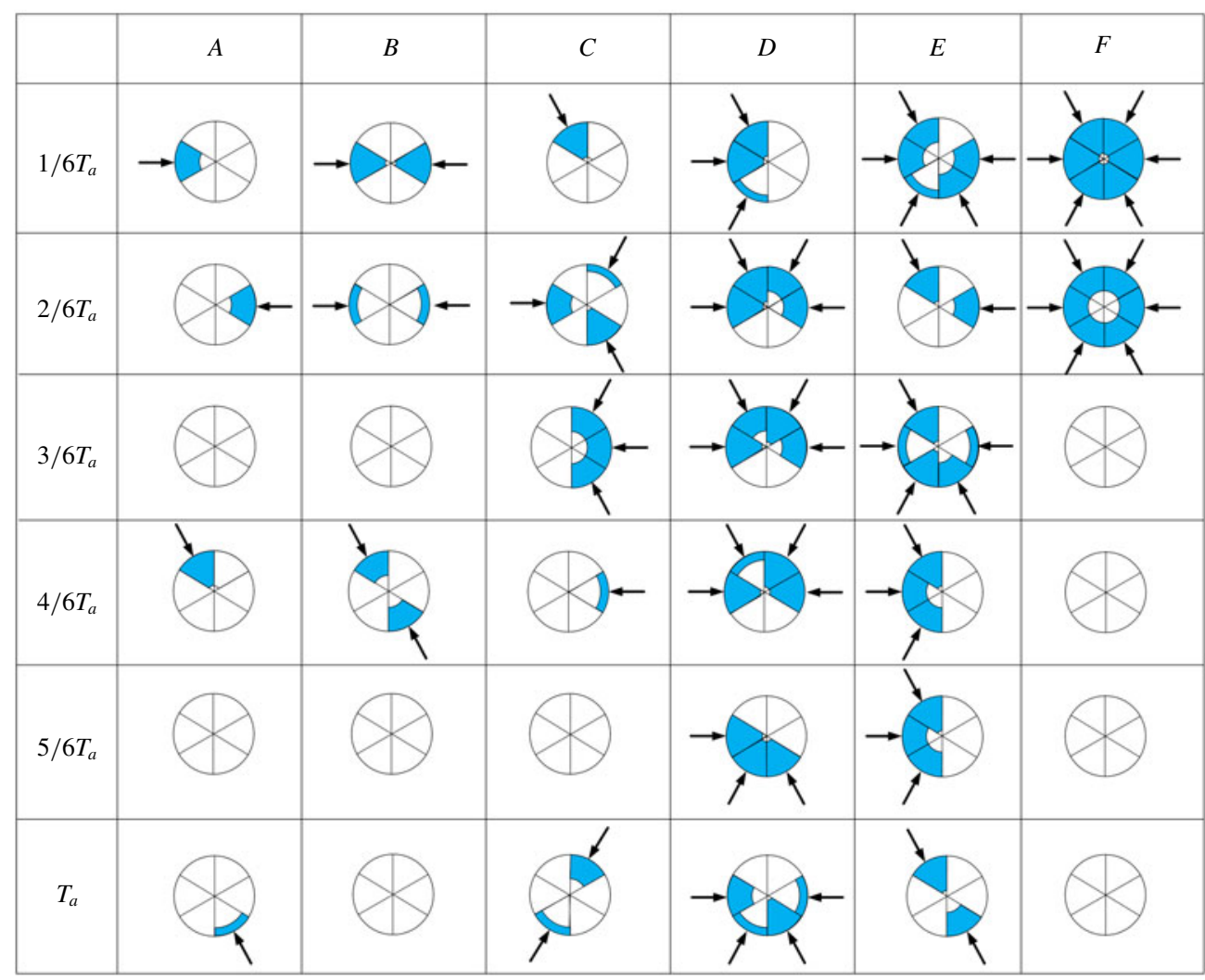

FIgURE 13. Actuation associated with control laws $A-F$, extracted from figure 12(a). The symbols are as in figure 9.

where $\mathrm{Co}_{12}$ and $Q_{12}$ represent the cospectrum and quadrature spectrum of $u_{1}$ and $u_{2}$, respectively (Zhou, Zhang \& Yiu 2002).

An even larger lateral spread is achieved for helical forcing at $n=2$ (figure $11 \mathrm{~h}$ ) due to the rotating flow structure (figure 10h1-h6). The $E_{u}$ measured on the centreline shows less pronounced peaks at $f / f_{a}=1.0$ and its harmonics than its counterpart for axisymmetric forcing. This is because there are only two or three injecting minijets at any instant for helical forcing (figure $9 h 1-h 6$ ), which produce considerably less velocity fluctuations at the jet centre than six minijets (figures 5 and 6). Furthermore, the peaks at $f / f_{a} \geqslant 2.0$ disappear at $x / D \geqslant 1$. The $\Phi_{12}$ is about $\pi$ over a range of frequencies about $f / f_{a}=1.0$ (figure $15 h$ ), as is expected based on figure $9(h 1-h 6)$.

At $n=5$, the jet column wobbles right and left, as indicated by yellow arrows in figure $11(f 1)$, in the flapping plane (the $x-y$ plane) but not in the orthogonal $x-z$ plane (figure 11f2), which is symmetrical about $y=0$. The lateral spread appears exceeding appreciably that for helical forcing, echoing the considerably improved mixing shown in figure 8. Interestingly, the peaks at $f / f_{a}=1.0$ and its harmonics in $E_{u}$ are less pronounced than their counterparts of helical forcing (figure 14). The peaks of the former decay more rapidly, completely vanishing by $x / D=4.0$, than 

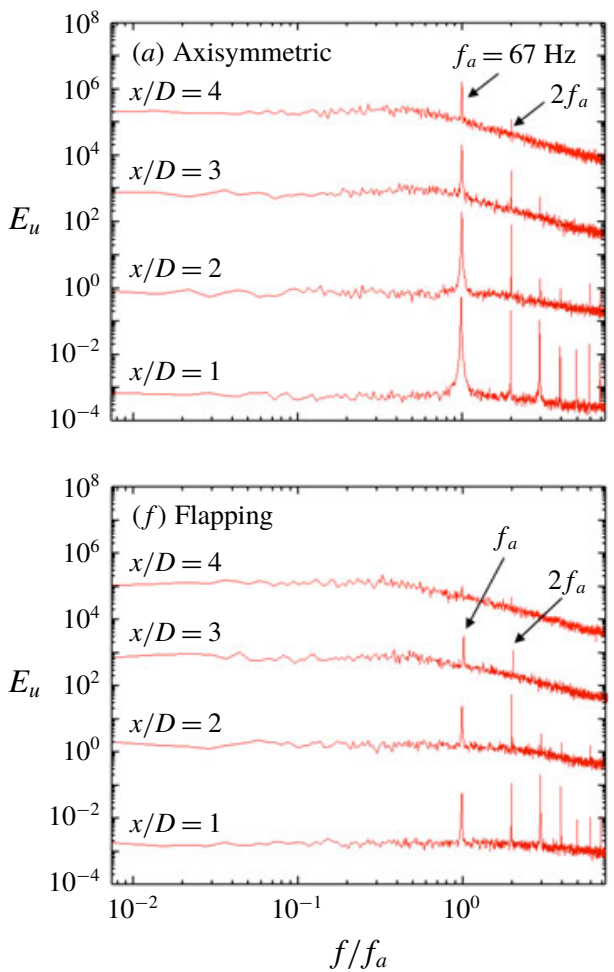
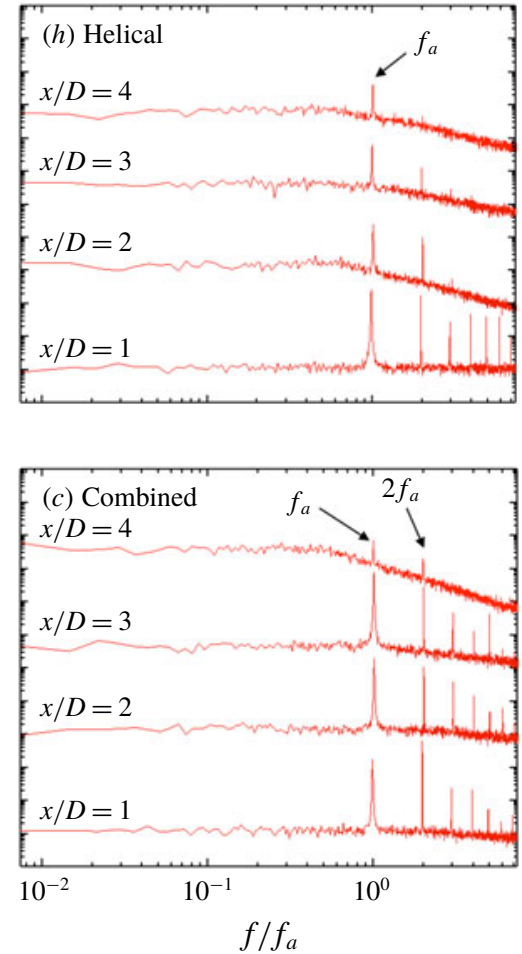

FIGURE 14. Normalized streamwise power spectra of fluctuating velocity $u$ measured on the centreline in the presence of the main jet: $(a)$ axisymmetric, $(h)$ helical, $(f)$ flapping and $(c)$ combined forcings.

the latter. The observation is consistent with the perception that it is the flapping motion, not the large-scale vortices, that plays a predominant role in enhancing mixing in this case. The flapping motion of the jet is characterized by a negative correlation between the two fluctuating streamwise velocities obtained on the opposite side of the jet (Goldschmidt \& Bradshaw 1973). Indeed, $\Phi_{12}$ is about $\pi$ over a very narrow frequency band about $f / f_{a}=1.0$ in the flapping plane but zero over a rather broad range of frequencies in the non-flapping plane (figure $15 f 1, f 2$ ), as observed by Yang \& Zhou (2016). Note that the peak at $f / f_{a}=2.0$ is larger than at $f / f_{a}=1.0$ for flapping forcing (figure 14f). This behaviour is ascribed to the flapping motion (figure $11 f 1$ ) caused by two separate excitations with a phase shift of $\pi$ within each excitation cycle (figure 9), which are captured by the hot wire.

The combined mode is distinct from all other forcings. Firstly, its spread shown in figure $11(c)$ is clearly the largest of all, due to the presence of both flapping and helical motions (figures $9 c 1-c 6$ and 10c1-c6), internally consistent with the smallest $J$ in figure 8. Secondly, its $E_{u}$ (figure 14c) displays a number of differences from other forcings. The peak at $f / f_{a}=1.0$ grows in amplitude from $x / D=1$ to 3 , while its counterpart for the other three forcings all decay quickly. Furthermore, the peaks at the higher harmonics of $f / f_{a}=1.0$ decay little for the same range of $x / D$, in distinct contrast to their counterparts of other forcings where these peaks retreat rapidly. Naturally, compared with the other three forcing modes, there are many peaks at the higher harmonics of $f / f_{a}=1.0$, especially at $x / D=3$. When manipulating the 

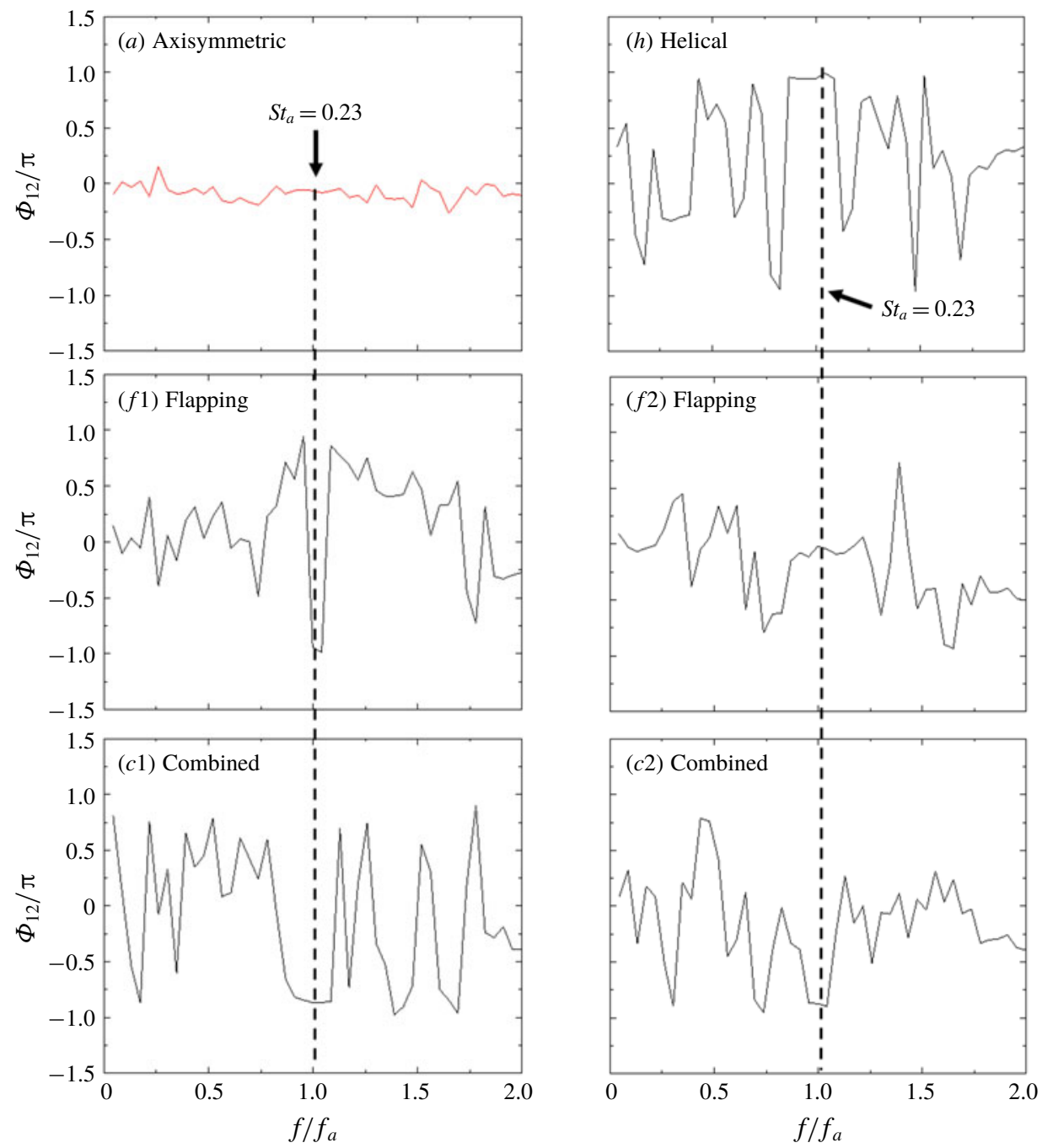

FIGURE 15. Phase spectrum $\Phi_{12}$ between two streamwise fluctuating velocity signals $u$ from hot wires measured at $x / D=1$ and $y / D$ or $z / D= \pm 0.3$. $S_{t a}=f_{a} D / \bar{U}_{j}$.

main jet using a single unsteady minijet, Perumal \& Zhou (2018) made a similar observation, which was ascribed to the use of a small duty cycle. The small duty cycle occurs in every phase of the combined mode (figure $9 c 1-c 6$ ). Another note is that the peak at $f / f_{a}=2.0$ is larger than at $f / f_{a}=1.0$ for $x / D=1$ and remains very pronounced downstream, similarly to the flapping forcing case (figure 14f). Thirdly, its $\Phi_{12}$ (figure $15 c$ ) approaches anti-phase at $f / f_{a}=1.0$ for both orthogonal planes examined. A rather broad plateau occurs at about $f / f_{a}=1.0$, where $\Phi_{12} \approx-0.86 \pi$. This phase shift differs appreciably in value from flapping or helical forcing where $\Phi_{12} \approx \pi$, and is probably connected to the presence of the oscillating component in this mode. Note that the combined mode produces the nearly anti-phased behavior for all planes through the $x$-axis. However, in flapping forcing, this anti-phased behavior takes place only in the flapping plane. 


\subsection{Momentums impinging upon the main jet and jet centre trajectory}

Additional insight may be gained into the flow physics of the combined mode by examining the sum of the momentums due to individual injecting minijets impinging upon the main jet and the direction of their resultant momentum. As the averaged mass flux is the same for all minijets, the maximum actuation velocity scales roughly with the inverse of the duty cycle $\alpha$, as demonstrated in figure 2. As such, the maximum actuation velocity $U_{a, i}$ of the $i$ th minijet is proportional to the product of $b_{i}(t)$ and $1 / \alpha_{i}$, viz.

$$
U_{a, i}(t) \propto \frac{b_{i}(t)}{\alpha_{i}},
$$

where $b_{i}(t)$ is a signal generated by $K_{i}(\boldsymbol{s}(t), \boldsymbol{h}(t))$, given in (4.1), its values 0 and 1 corresponding to the on- and off-states, respectively. The time-averaged mass flow rate is the same, $1.2 \%$, for every minijet. The sum of the momentums due to individual injecting minijets impinging upon the main jet is parameterized by

$$
A(t)=\sum_{i=1}^{N} \frac{b_{i}(t)}{\alpha_{i}} .
$$

By definition, the time-averaged amplitude is the number of active minijets, i.e. 0 for unforced flow and $\overline{A(t)}=N=6$ for actuation, implying a total time-averaged mass-flow rate of $7.2 \%$. On the other hand, the resultant momentum vector of the momentums associated with individual injecting minijets is given by

$$
\boldsymbol{Q}(t)=\sum_{i=1}^{N} \frac{b_{i}(t)}{\alpha_{i}} \boldsymbol{e}_{i},
$$

where $\boldsymbol{e}_{i}=-\left(\cos \theta_{i}, \sin \theta_{i}\right)$ is a unit vector in the direction of the $i$ th minijet located at angle $\theta_{i}$ in the $y-z$ plane. The minus sign refers to an inward velocity towards the jet centre. Here $N$ is the number of injecting minijets.

Figure 16 shows the variation in angle $\theta(t)$ of $\boldsymbol{Q}(t)$, with respect to the $y$-axis, and $A(t)$ with time $t$ over two actuation periods $T_{a}$ for the axisymmetric, helical, flapping and combined forcing modes $(n=1,2,5$ and 11). For axisymmetric forcing, $\theta(t)$ is undetermined as $\boldsymbol{Q}(t)$ vanishes identically, as $b_{1}=b_{2}=\cdots=b_{6}$ and $\sum \boldsymbol{e}_{i}=0$ (figure $9 a 1$ ). Therefore, $A(t)$, albeit large (figure 16a2), would not make the jet column oscillate (figure $11 a$ ). In the case of helical forcing, $\theta$ varies essentially linearly with $t$, as indicated by the red dashed line (figure 16h1). The stepwise behaviour is caused by the discontinuous on-off actuation $b_{i}(t)$. A large $A(t)$ or one half of the strength of axisymmetric forcing (figure $16 a 2, f 2$ ) occurs at $t / T_{a}=0.2$ and 0.7 for flapping forcing, which correspond to a phase shift of $\pi$ (figure 16f1). The behaviours of both $A(t)$ and $\theta(t)$ are fully consistent with our understanding of axisymmetric, helical and flapping forcings, thus providing a validation for applying $A(t)$ and $\theta(t)$ to describe the forcing on the main jet.

For the combined mode, the variations in both $A(t)$ and $\theta(t)$ with $t / T_{a}$ are more complicated. Nevertheless, a number of features can be identified. Firstly, after reaching the first maximum, as highlighted by a circle, $\theta(t)$ decreases, albeit not monotonically, over a duration of $1.3 \pi$ (figure $16 c 1$ ), similarly to helical forcing, as indicated by the red arrow. This feature implies a swirling forcing on the main jet. Secondly, the maxima of A exceed those of helical forcing, suggesting a stronger 

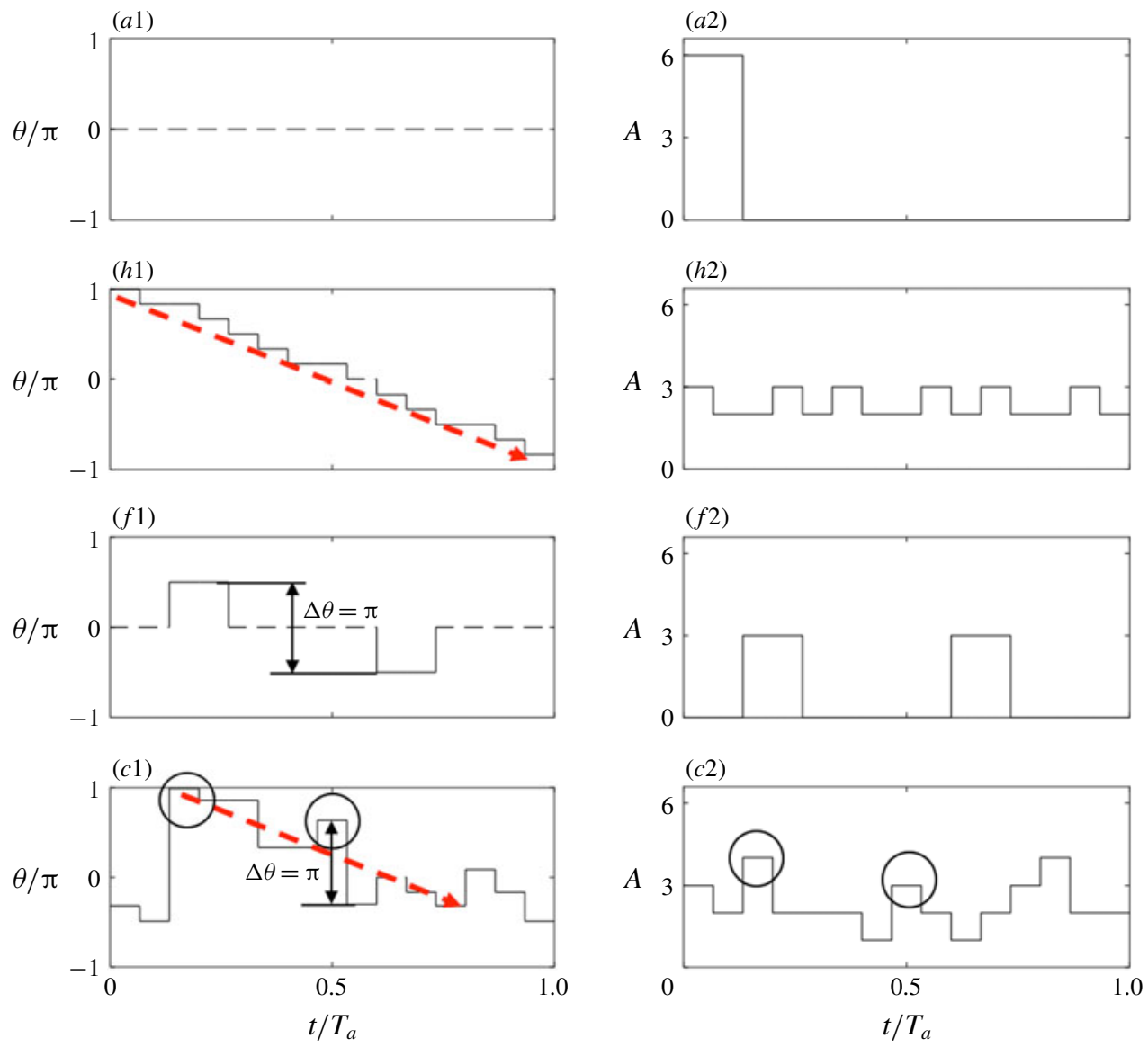

FIGURE 16. The angle $\theta(a 1, h 1, f 1, c 1)$ and magnitude $A(t)(a 2, h 2, f 2, c 2)$ of the effective minijet actuation vector $\boldsymbol{Q}(t)$ for the best control law of $n=1,2,5$ and 11 .

swirl actuation. Thirdly, the phase shift between the second local maximum $\theta(t)$ and a local minimum $\theta(t)$ at $t / T_{a}=0.67$ is $\pi$, as highlighted by the vertical arrow in figure 16(c1), pointing to the signature of flapping forcing. The local maximum $A$ at $t / T_{a}=0.67$ (figure $16 c 2$ ) is associated with a phase change of $\pi$ (figure $16 c 1$ ), highlighted by a circle. All the observations suggest that the combined mode is rather unique, featured by both helical and flapping motions, and further by the stronger strengths of flapping and vortical motions than other cases, thus accounting for the largest entrainment and mixing of all.

Evidently, the jet centre $\boldsymbol{r}_{c}=\left(y_{c}, z_{c}\right)$ responds to $\boldsymbol{Q}\left(t_{i}\right)(6.3)$, and it is plausible to that assume the jet centre trajectory is correlated with $\boldsymbol{Q}\left(t_{i}\right)$. This centre can be characterized as the 'centre of gravity' of the streamwise velocity in a cross-stream plane of $x=$ const.:

$$
\boldsymbol{r}_{c}(t)=\iint \mathrm{d} \boldsymbol{r} \boldsymbol{r} u(\boldsymbol{r}, t) / \iint \mathrm{d} \boldsymbol{r} u(\boldsymbol{r}, t) .
$$

Here, $\boldsymbol{r}=(y, z)$ represents the coordinate in the cross-stream plane. For simplicity, the $x$-dependency of $\left(y_{c}, z_{c}\right)$ will be dropped out hereafter. Over one excitation period 
with a time step $\Delta t=T / 6$, the jet centre takes six positions, i.e. $\boldsymbol{r}_{c}\left(t_{i}\right)=\left(y_{c}\left(t_{i}\right), z_{c}\left(t_{i}\right)\right)$, $t_{i}=i \triangle t, i=0,1, \ldots, 5$. We make the most simple assumption that the jet centre displacement $\left(\Delta y_{c}, \Delta z_{c}\right)\left(t_{i}\right)=\left(y_{c}\left(t_{i+1}\right)-y_{c}\left(t_{i}\right), z_{c}\left(t_{i+1}\right)-z_{c}\left(t_{i}\right)\right)$ over $\Delta t$ is proportional to the actuation momentum, viz.

$$
\boldsymbol{r}_{c}\left(t_{i+1}\right)=\boldsymbol{r}_{c}\left(t_{i}\right)+\boldsymbol{Q}\left(t_{i}\right)
$$

for $i=0, \ldots, 5$, where the proportionality constant is set to unity again for simplicity. The discrete time dynamics (6.5) can be considered to be a rough discretization of the dynamics equation $\mathrm{d}\left(\boldsymbol{r}_{c}\right) / \mathrm{d} t=\boldsymbol{Q}$, which describes the jet centre motion under the external momentum. Equation (6.5) represents 10 equations for 12 unknown jet centre coordinates. The remaining equations are obtained from the observation that the control law is periodic in time and the time-averaged actuation momentum vanishes based on experimental constraints, i.e. the same averaged mass flow through each minijet. Hence, the average jet centre position can be expected to vanish:

$$
\sum_{i=0}^{5} \boldsymbol{r}_{c}\left(t_{i}\right)=0 \text {. }
$$

Equations (6.5) and (6.6) constitute 12 linear equations for 12 unknowns, describing the motion of the jet centre over one excitation period. The jet centre dynamics is most easily solved by starting the integration at the origin with $x_{c}\left(t_{0}\right)=y_{c}\left(t_{0}\right)=0$, iteratively computing the positions at $t_{i}, i=1, \ldots, 5$ with (6.5) and adding a translation consistent with a vanishing averaged jet centre (6.6).

Figure 17 presents the trajectories of the jet centre within each excitation period for the four forcings, which are calculated based on the control laws shown in figure 9 or (A 1)-(A 3) and (6.5)-(6.6). Apparently, the jet centre vanishes identically for axisymmetric forcing where $\boldsymbol{Q}\left(t_{i}\right) \equiv 0$ and oscillates along the $y$-direction between two extremes in the $x-y$ plane for flapping forcing, where $\boldsymbol{Q}\left(t_{i}\right)$ changes from the positive $y$-direction at one phase to the negative at the next phase or vice versa. Helical forcing, i.e. a uniformly rotating $\boldsymbol{Q}$ vector, leads to a uniformly precessing jet and the jet centre moves along a circle around the axis of symmetry. The result conforms to previous reports. Koenig et al. (2016) experimentally investigated the turbulent jet under the helical mode excitation and observed a precessing jet column when the helical structures were spatially amplified in the shear layer. Zhang \& Turner (2016) found in a similar experiment that the jet centreline under helical excitation was offset slightly and precessed around the initial axis of the core flow. For the combined forcing, the motion of the jet centre is more complicated. Its trajectory is apparently ellipse-like, suggesting the occurrence of a precession jet. In contrast to helical forcing, the distance of the jet centre from the centre of symmetry varies, along with the separation between the centres of two consecutive phases, suggesting the speed of swirling changes with time. Furthermore, this ellipse-like path indicates an oscillating jet column, a feature of the flapping motion.

\subsection{Velocity field}

The velocity field may provide us with the crucial information on the flow structure. Figure 18 presents the radial profiles of the hot wire measured $\bar{U} / \bar{U}_{j}$ at $x / D=0.05$. A number of observations could be made. Firstly, for all controlled cases except helical forcing, the mean velocity profile displays an overshoot at the nozzle exit, as 


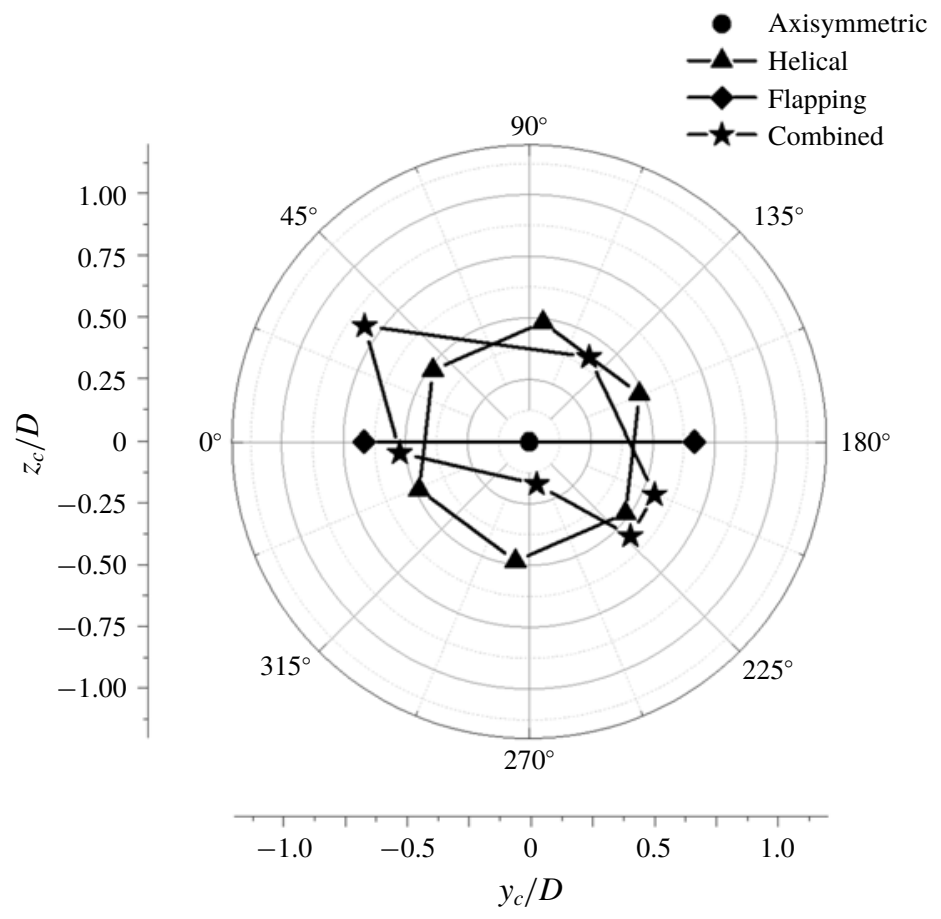

FIGURE 17. Jet centre trajectory $\left(y_{c}, z_{c}\right)$ predicted from the resultant momentum vector $\boldsymbol{Q}\left(t_{i}\right)$ of the momentums due to individual injecting minijets at phases $t_{i}=i T_{a} / 6(i=$ $1,2, \ldots, 6)$.

noted by Wu et al. (2018a) who used a single unsteady minijet to manipulate the main jet. Andreopoulos \& Rodi (1984) made the same experimental observation in case of a circular jet in a cross-stream. They explained that the cross-stream fluid acted like a partial cover over the jet exit, causing the jet flow to bend around and to accelerate so that the velocity of the bent-over jet was somewhat higher than the cross-stream velocity. Secondly, the widths of the mean velocity profiles under control are larger than that of the unforced jet, indicating that the shear layer grows laterally, and the main jet becomes wider right at the nozzle exit. Thirdly, the mean velocity profiles of the axisymmetric and helical forcing at the jet exit are almost symmetrical about $y / D$ or $z / D=0$, in general with its maximum at the centre (figure $18 a, h$ ). The velocity profile of flapping forcing is also symmetric about $y / D$ and $z / D=0$ (figure 18f), though displaying two peaks off the centre in the $x-y$ or flapping plane and indicating the occurrence of bifurcation. As shown in figure 11( $f 1)$, two consecutive rings are locally connected, forming a zigzag flow structure, as observed in Carlos \& Olivier's (2002) numerical investigation of a bifurcating jet (their figure $4 a$ ). Furthermore, the cross-flow distributions $\bar{U} / \bar{U}_{j}$ at $x / D=3-5$ (figure 18f) display two peaks in the bifurcation $(x-y)$ plane, while those in the bisection $(x-z)$ plane show only one peak on the centreline. Also, the jet grows slowly in width in the bisection plane. All the features are similar to Lee \& Reynolds's (1985) data where loudspeakers were used to produce a bifurcating water jet at $R e_{D}=4300$. The present data deviate quantitatively from Lee \& Reynolds (1985) as a result of distinct actuation techniques, experimental setups and $R e_{D}$ between the two investigations. Finally, the velocity profile under the 


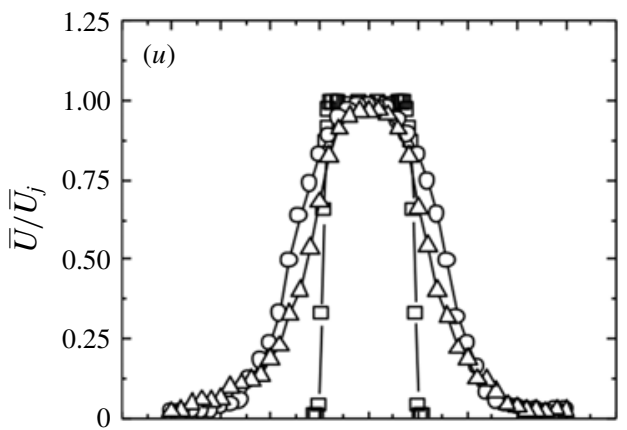

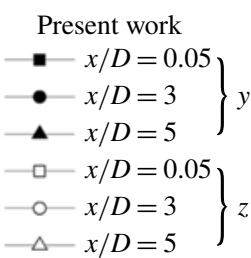

Lee \& Reynolds (1985)

$\star x / D=6.5 y$

๘ $x / D=6.5 z$
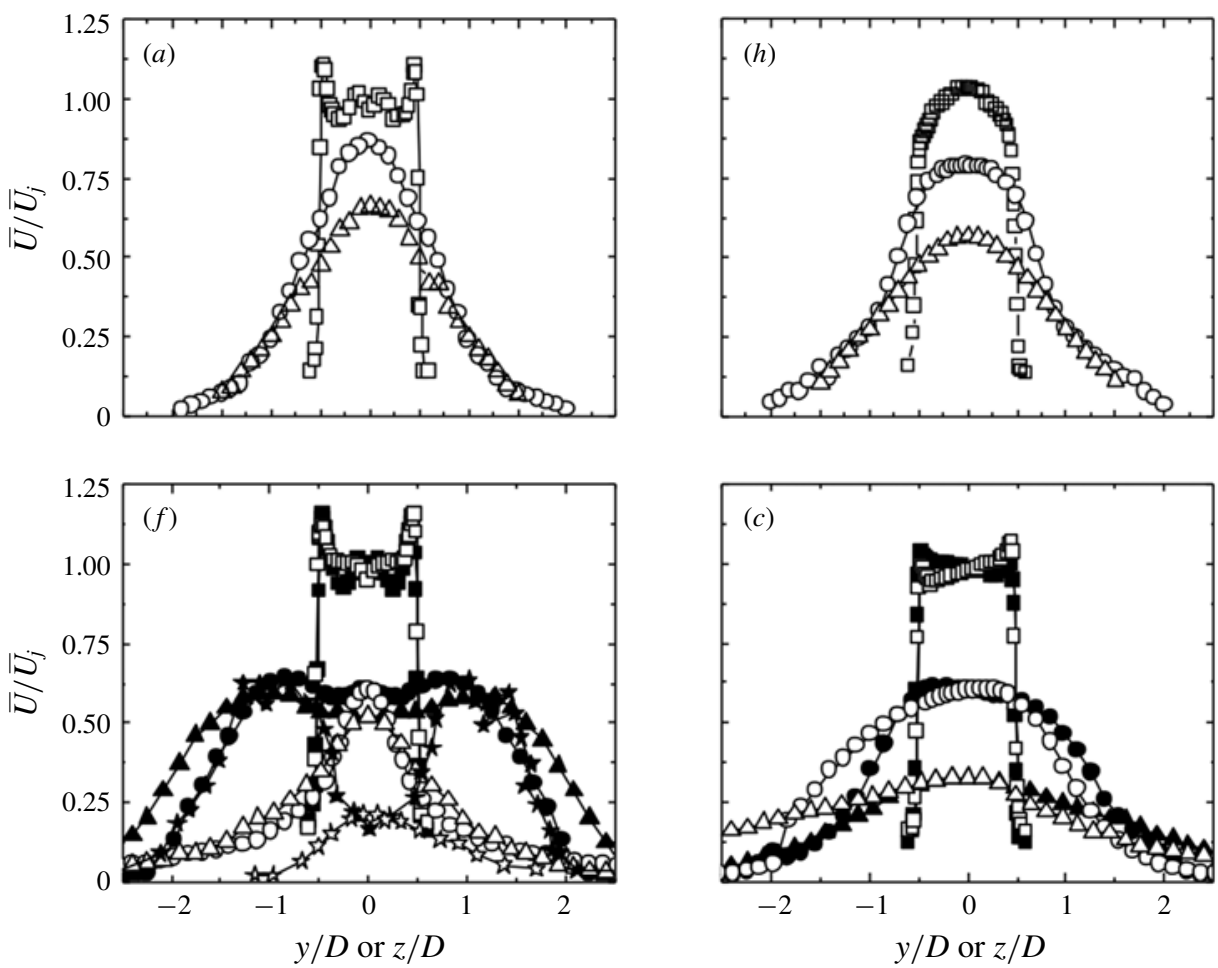

FIGURE 18. Radial distributions of $\bar{U} / \bar{U}_{j}$ measured at different $x / D$ in both $x-z$ and $x-y$ planes for $(u)$ unforced jet; $(a)$ axisymmetric; $(h)$ helical; $(f)$ flapping, the bifurcation jet of Lee \& Reynolds (1985) is included for comparison; (c) combined.

combined forcing mode (figure $18 \mathrm{c}$ ) is distinct from others and a little tilted at the nozzle exit. Further downstream, $\bar{U} / \bar{U}_{j}$ remains asymmetrical about the jet centre but, unlike the flapping mode (figure $18 f$ ), does not show the twin-peak distribution. Wong et al. (2003) produced a precession jet by issuing a jet into a cylindrical chamber with a small axisymmetric inlet at one end and an exit lip at the other. The inlet flow separates at the abrupt inlet expansion and reattaches asymmetrically to the wall of the chamber. Asymmetry of the flow within the chamber causes the reattaching flow to precess around the inner wall of the chamber, resulting in a precessing exit flow. An asymmetric and rotating pressure field is thus established so that the entire flow field, including the emerging jet, precesses (Nathan, Hill \& Luxton 1998). 

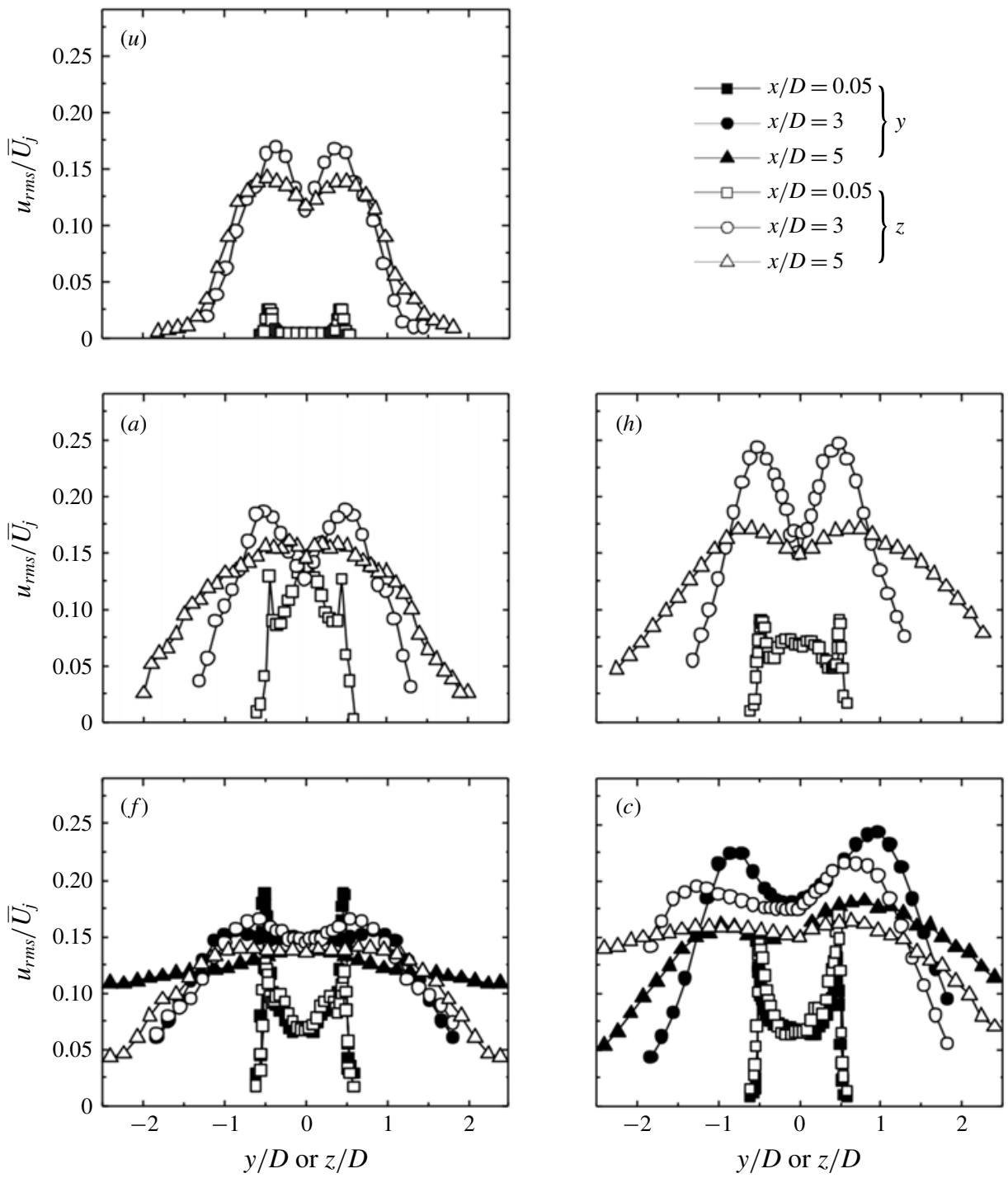

FIgURE 19. Radial distributions of $u_{r m s} / \bar{U}_{j}$ measured at different $x / D$ in both $x-z$ and $x-y$ planes for $(u)$ unforced jet; $(a)$ axisymmetric; $(h)$ helical; $(f)$ flapping; $(c)$ combined.

The precessing jet proves to be highly effective in increasing the near-field spreading. Interestingly, the present distributions of $\bar{U} / \bar{U}_{j}$ exhibit a similarity to their counterparts of the precession jet (please refer to figure 10 in Wong et al. (2003)) at the nozzle exit and downstream development.

The radial distributions of $u_{r m s} / \bar{U}_{j}$ (figure 19) are reasonably symmetrical about $y / D$ or $z / D=0$ under control, except under combined forcing. The $u_{r m s}$ rises greatly at $x / D=0.05$ throughout the main jet, as compared to the uncontrolled jet (figure $19 u$ ) where $u_{r m s} / \bar{U}_{j}=0.3 \%$ at $y / D=0$ and $2.5 \%$ at $y / D \approx \pm 0.43$ due to the shear layer. The peak in the shear layer becomes very pronounced. The result suggests a turbulent jet at the nozzle exit, which is internally consistent with flow visualization data (figure 11), and the shear-layer instabilities are significantly amplified. The $u_{r m s} / \bar{U}_{j}$ 
for axisymmetric forcing in the centre region is largest of all, due to the simultaneous injection of six minijets into the main jet, which causes a strong disturbance in the central region (figure 19a). The $u_{r m s} / \bar{U}_{j}$ distribution displays twin peaks for helical forcing (figure 19h). This is reasonable as helical forcing may produce a hurricane-like helical motion with a centre that is more stable than the surrounding motion. The $u_{r m s} / \bar{U}_{j}$ at $x / D=0.05$ under flapping forcing in the $x-y$ plane is larger than in the $x-z$ plane in the shear layer (figure 19f), as observed by Hussain \& Husain (1989) and Zaman (1996). The twin-peak behavior is evident along the $z$-axis due to the flapping motion, but not so along the $y$-axis. The $u_{r m s} / \bar{U}_{j}$ distributions under combined forcing are asymmetrical about the centre at the jet exit (figure 19c), and again resemble the precession jet (Mi \& Nathan 2005). Unlike the case under flapping forcing, the $u_{r m s} / \bar{U}_{j}$ profiles under combined forcing display marked twin peaks along the $y$ and $z$ axes, where the right peak is more pronounced than the left, which is probably linked to the helical motion. Furthermore, the $u_{r m s} / \bar{U}_{j}$ peaks at $x / D=3$ are substantially higher along the $y$-axis than along the $z$-axis, which remains discernible at $x / D=5$. All the features have been observed in the precession jet. Mi \& Nathan (2005) investigated the streamwise development of $u_{r m s}$ in a precession jet. As shown in their figure 18, the $u_{r m s}$ of the precessing jet is asymmetric compared with a non-precession jet and exhibits two peaks, located tangentially 'in front of' and 'behind' the jet centre. The 'front' peak is more pronounced than the 'rear' peak. The similar behaviours between the jet under combined forcing and a precessing jet may suggest that the so-called combined forcing may have produced a precession jet. This suggestion is further corroborated by the downstream development of the centreline mean and fluctuating velocities $\bar{U}_{c l} / \bar{U}_{j}$ and $u_{c l, r m s} / \bar{U}_{j}$ presented below.

The variations in $\bar{U}_{c l} / \bar{U}_{j}, u_{c l, r m s} / \bar{U}_{j}$ and $\bar{U}_{j} / \bar{U}_{c l}$ are presented in figure 20 for various forcing modes as well as the unforced jet. It is worth pointing out that our contraction nozzle is extended by a $47 \mathrm{~mm}$ long smooth tube of the same diameter as the nozzle exit $D$ where the minijet assembly is mounted. In spite of this difference, the unforced jet displays the well-known features. Firstly, $f_{0} D / \bar{U}_{j}$ is 0.45 , falling in the expected range 0.24-0.64 (e.g. Gutmark \& Ho 1983; Zhou et al. 2012). Secondly, $\bar{U}_{c l} / \bar{U}_{j}$ and $\bar{U}_{j} / \bar{U}_{c l}$ (figure $20 a, c$ ) agree both qualitatively and quantitatively with Mi \& Nathan (2005) $\left(R e_{D}=8050\right)$ and Seidel et al.'s (2005) $\left(R e_{D}=8800\right)$ measurements. Following Todde, Spazzini \& Sandberg (2009), we may fit the data to $\bar{U}_{c l} / \bar{U}_{j}=B\left[\left(x-x_{0}\right) / D\right]^{-1}$, where $x_{0}$ and $B$ denote the virtual origin and decay constant, respectively. Then, $x_{0} / D=-0.49$ and $\bar{U}_{c l} / \bar{U}_{j}$ decays at a rate of $x^{-1}$ beyond $x / D=5$, as reported by previous investigations (e.g. Mi, Nobes \& Nathan 2001). Thirdly, the streamwise distribution of $u_{c l, r m s}$ exhibits one peak at $x / D=3.5$ and another at $x / D=7.5$ (figure $20 b$ ). The former is connected to the breakdown of the primary ring vortices, and the latter is due to early transition to turbulence $(\mathrm{Mi}, \mathrm{Xu}$ \& Zhou 2013). Similar observations were made by Todde et al. (2009) (their figure 6 ) and by Mi et al. (2013), whose data are included in figure 20(b). Departures between the present and other's data are not unexpected in view of differences in, inter alia, experimental setup and $R e_{D}$ among the investigations. The potential core length of the unforced jet is approximately $5 D$, beyond which $\bar{U}_{c l}$ appears dropping approximately linearly. Note that $\bar{U}_{c l} / \bar{U}_{j}$ for axisymmetric forcing exceeds 1.0 given $x / D \leqslant 2$, higher than those of the other modes. This is due to an increase in the mass flow rate by $7.2 \%$. A similar observation is made by Seidel et al. (2005) who observed, with 16 minijets blowing, an increase in the centreline mean velocity near the jet exit. Under all control modes, $\bar{U}_{c l} / \bar{U}_{j}$ decays rapidly right from the beginning. The minijet actuation reduces $\bar{U}_{c l} / \bar{U}_{j}$ significantly at $x / D \leqslant 8$ (figure $20 a$ ), 

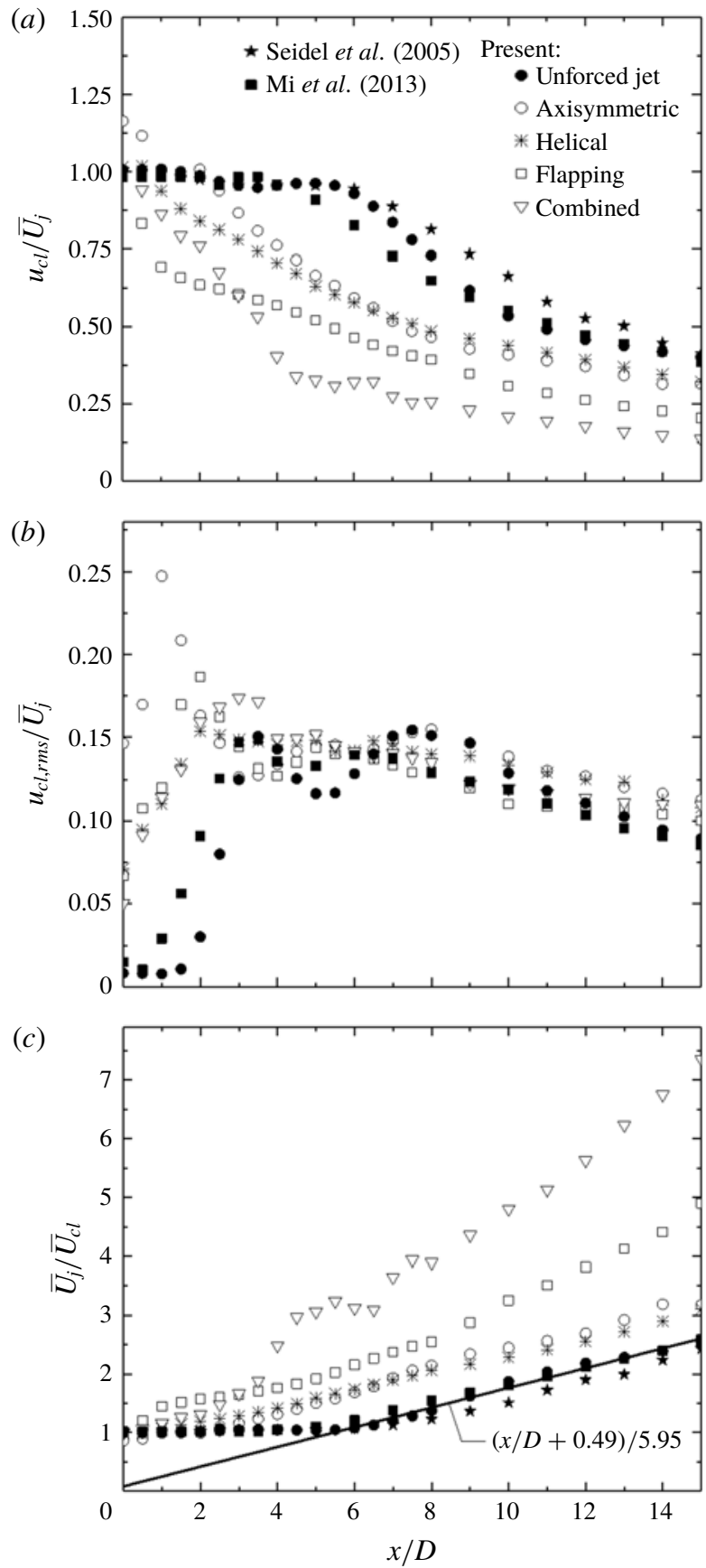

FIgURE 20. Streamwise variations of hot-wire measured centreline mean and r.m.s. velocities: (a) $\bar{U}_{c l} / \bar{U}_{j},(b) u_{c l, r m s} / \bar{U}_{j}$, (c) $\bar{U}_{j} / \bar{U}_{c l}$.

demonstrating the efficacy of minijet injections. This efficacy can be attributed to the earlier increase in the turbulence levels when the radial injections are present; a large turbulence level (figure 20b) is correlated with a rapid drop in $\bar{U}_{c l} / \bar{U}_{j}$ (figure $20 a$ ). 
Interestingly, the variations in $u_{c l, r m s} / \bar{U}_{j}$ for helical and combined forcings are quite similar to each other, growing almost linearly first and then experiencing a small drop before fluctuating slightly around 0.15 (figure 20b). This similarity is ascribed to the common feature of the two control modes, i.e. the swirling motion. However, it is combined forcing that maintains the rapid and linear growth further downstream, up to $x / D=3$, and retreats little by $x / D=3.5$. It is noted earlier in figure $14(c)$ that those peaks at $f / f_{a}=1,2,3,4$ and 5 in $E_{u}$ also remain pronounced up to $x / D=3$. This coincidence suggests that the excited coherent structures account for the linear growth in $u_{c l, r m s} / \bar{U}_{j}$ (figure $20 b$ ) and the rapid decay in $\bar{U}_{c l} / \bar{U}_{j}$ (figure $20 a$ ). In contrast, the rapid growth of $u_{c l, r m s} / \bar{U}_{j}$ is only up to $x / D=2$ for helical forcing and even only up to 1 for axisymmetric and flapping forcings (figure $20 b$ ). As such, $\bar{U}_{c l} / \bar{U}_{j}$ keeps decaying rapidly and almost linearly until $x / D=4.5$ for the combined forcing mode and remains well below other cases further downstream (figure 20a). It is worth pointing out that the precessing jet is also characterized by a substantially faster decay than the non-precessing jet (Mi \& Nathan 2005).

Jet spreading rate and the overall entrainment rate may be well quantified by the downstream variation of the jet width (e.g. Zhou et al. 2012). Following Hussain $\&$ Husain (1989), we define an equivalent jet width by $R_{e q}=\left[R_{H} R_{V}\right]^{0.5}$, where $R_{H}$ and $R_{V}$ denote the mean-velocity half-widths in the $x-z$ and $x-y$ planes, respectively. The half-width is defined as the distance between the jet centreline and the location at which $\bar{U}=0.5 \bar{U}_{c l}$. Figure 21 shows the downstream evolution of $R_{V}, R_{H}$ and $R_{e q}$. While changing little for the unforced jet, $R_{V}, R_{H}$ and $R_{e q}$ grow appreciably in the controlled jet. Evidently, $R_{e q}$ is the largest for the combined mode, followed by flapping, helical and axisymmetric, though the latter two do not differ much. The results provide additional support for our choice of $J$ as a measure for the mixing efficacy.

To understand further the predominant flow structures under the four forcings, in figure 22 we examine typical instantaneous $V$ - or $W$-contours, measured using PIV, in the $x-y$ and $x-z$ planes. In the unforced jet, the positive and negative velocity concentrations occur in pair and are mirrored by another pair, though with swapped signs, on the other side of the centreline (figure 22u). Apparently, the two pairs of velocity concentrations are associated with the two vortical structures, as indicated by symbols ' + ' and ' $x$ ', of one ring vortex. Axisymmetric forcing leads to the topologically unchanged velocity contours but a significantly increased size in the velocity concentrations which now start to occur in the near proximity of the nozzle exit (figure 22a). Under helical forcing, the vortical structures above and below the centreline are stagger-arranged and their same-signed velocity contours are connected, forming inclined strips alternately in sign (figure 22h). Under flapping forcing (figure $22 f 1$ ), the $V$-contours in the $x-y$ plane exhibit alternate upward and downward motions which are absent in the $W$-contours in the $x-z$ plane. Some interesting observations can be made for combined forcing. Firstly, the inclined strips of velocity contours, alternately in sign, are seen in both planes (figure 22c1,c2). Secondly, the velocity concentrations reach in general the maximum contour level of \pm 0.5 , greater than those associated with other forcings $( \pm 0.4)$, indicating a stronger spread/entrainment or mixing. Thirdly, the pattern of velocity contours and their maximum strength persist considerably further downstream, reaching $x / D \approx 5$, whilst the upward and downward motions under flapping forcing disappear at $x / D \approx 3$, suggesting a prolonged entrainment and more thorough mixing. The observation is again internally consistent with the rapid drop until $x / D \approx 4.5$ in the centreline mean velocity decay (figure 20a). 

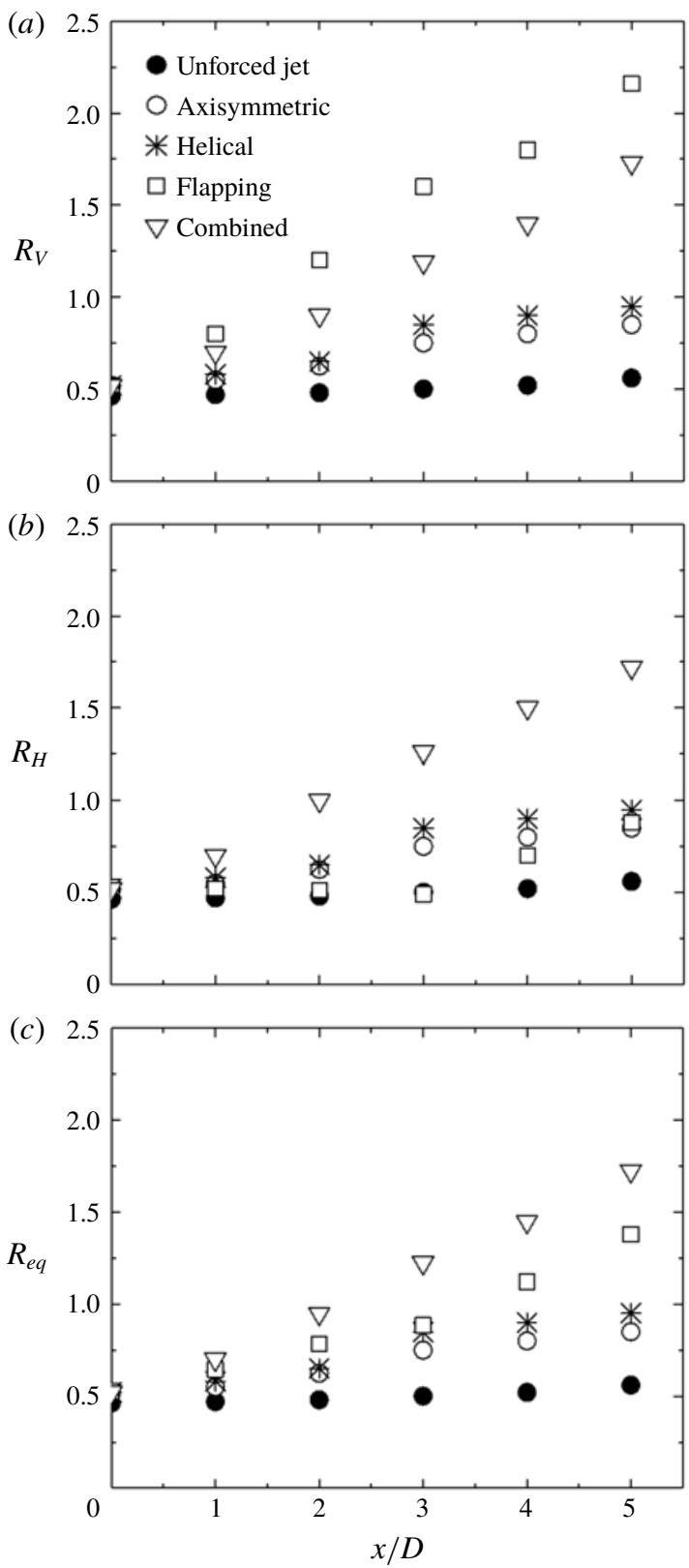

FIGURE 21. Mean-velocity half-widths at different $x / D$ : $(a) R_{V}$ in the $x-y$ plane, $(b) R_{H}$ in the $x-z$ plane and $(c)$ the equivalent half-radius $R_{e q}$.

More insight may be gained into the predominant flow structures under control by examining typical instantaneous velocity vectors superimposed with the corresponding isocontours of streamwise vorticity $\omega_{x}^{\star}=\omega_{x} D / \bar{U}_{j}$ in the $y-z$ plane at $x / D=0.25$ (figure 23). Under axisymmetric forcing (figure $23 a 1, a 2$ ), the vectors show the inward or outward motions associated with the ring-like structures, which are axisymmetric and highly repeatable. There are six pairs of counter-rotating $\omega_{x}^{\star}$ concentrations 

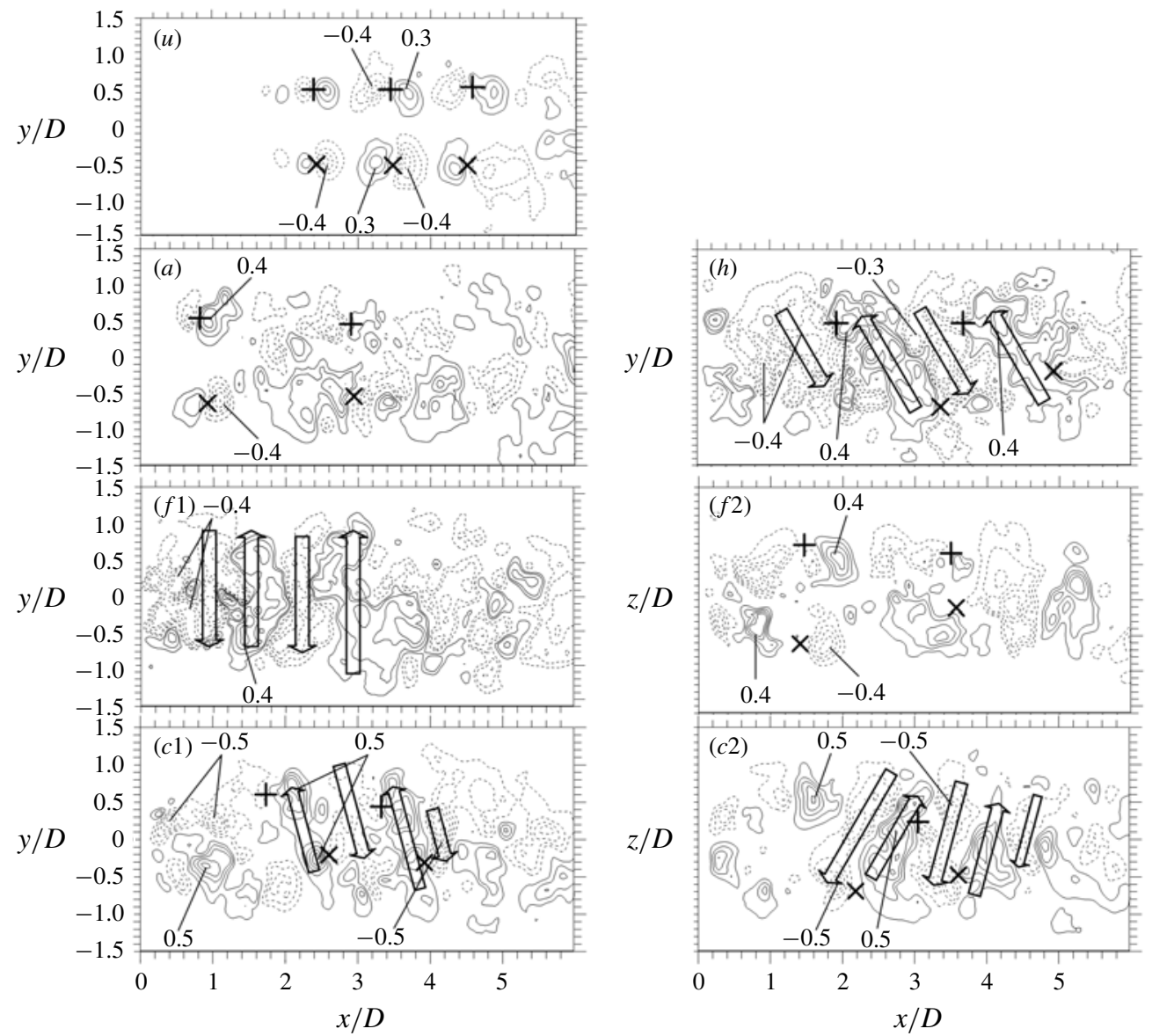

FIGURE 22. Isocontours of typical instantaneous velocities $V / \bar{U}_{j}$ or $W / \bar{U}_{j}$ in the $x-y$ and $x-z$ planes: $(u)$ unforced jet, $(a)$ axisymmetric, $(h)$ helical, $(f)$ flapping and $(c)$ combined forcing. Contour interval $=0.1$. The lowest contour level is 0.1 for all plots. Solid and broken contours represent the positive (upward motion) and the negative (downward motion), respectively. Symbols ' + ' and ' $x$ ' denote anti-clockwise and clockwise vortices, respectively. The arrows indicate the moving direction of the fluid slice.

arranged about the centre (figure 23a), apparently generated by the six axisymmetrically placed minijets. The rotational motion under helical forcing is evident and the fluid moves inward along the circumference (figure 23h1,h2). The core region appears rather stagnant. The phase of injecting minijets is clockwise incremented by $60^{\circ}$ (figure 9h), producing a corkscrew type of structure (figure 10h1-h6), as shown by Koch et al. (1989). Figure 23( $f 1, f 2)$ shows the cross-flow motion that switches from one direction at one moment to the opposite at another under flapping forcing, accompanied by one pair of counter-rotating $\omega_{x}^{\star}$ concentrations, as shown by Yang \& Zhou (2016). The velocity vectors in figure $23(c 1, c 2)$ exhibit the clockwise rotational motion under combined forcing. The area of rotational motion, as indicated by the arrows in figure 23(c1), is in general larger than that under helical forcing (figure 23h1-h2). Note that ambient fluid may be entrained into the jet core area from various circumferential locations, e.g. the upper left and lower right corners, 

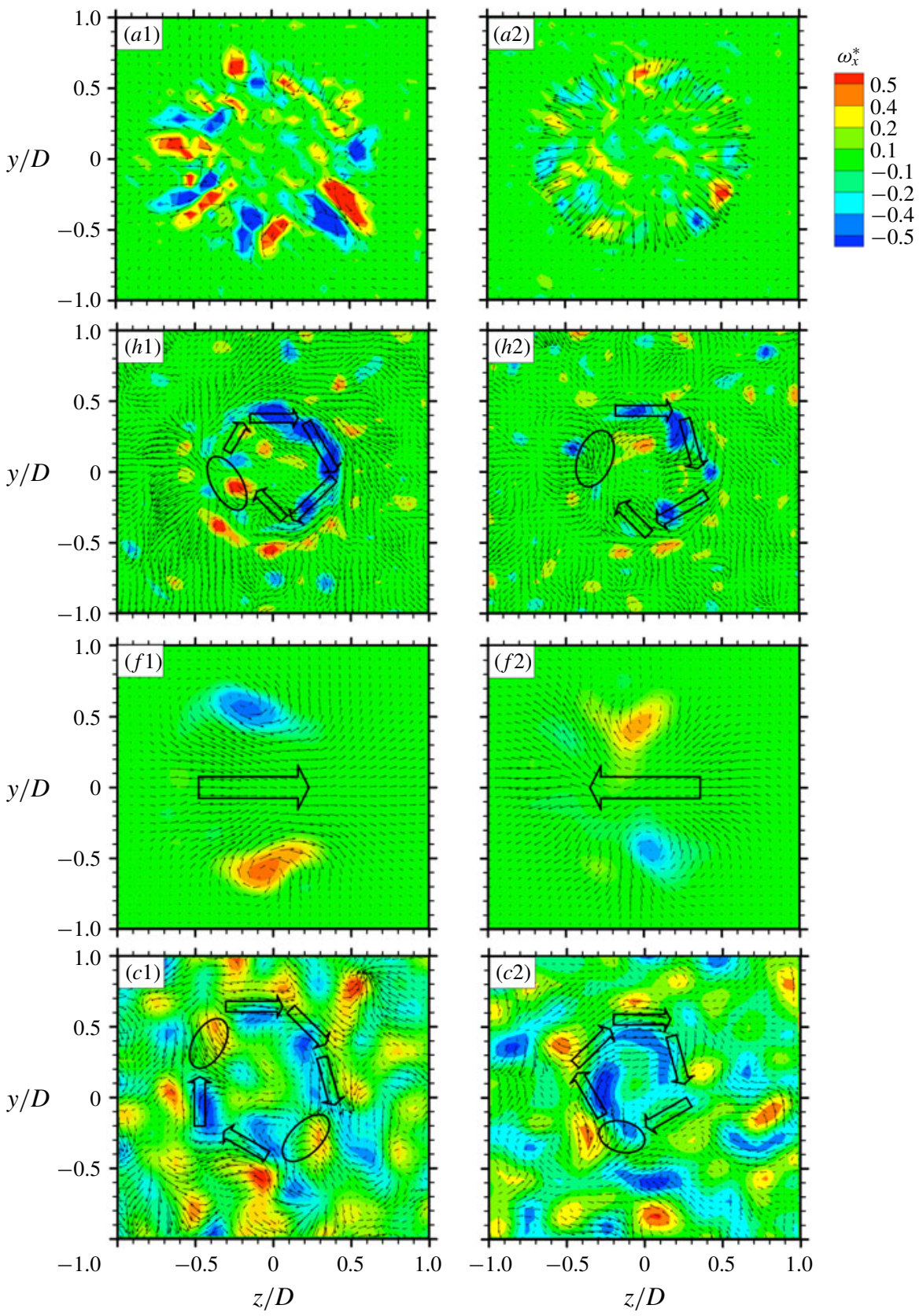

FIgURE 23. The typical PIV snapshots of instantaneous velocity vectors in a cross-sectional plane of $(u)$ unforced jet, $(a)$ axisymmetric, $(h)$ helical, $(f)$ flapping and $(c)$ combined forcings at $x / D=0.25$. The open arrow represents the motion of the jet fluid. The elliptic contour indicates where fluid is entrained into the jet core area.

as indicated by the elliptic contours in figure 23(c1), while under helical forcing ambient fluid comes into the core area largely from only one location, as highlighted by the elliptic contours (figure 23h1,h2). Furthermore, there are many vorticity 
concentrations of both signs in figure 23(c1-c2). The core area is dominated by the vorticity concentrations of negative sign, while the region surrounding the core is populated with those of both signs. This is very different from flapping forcing where there is only one pair of opposite-signed vorticity concentrations. This is also markedly different from helical forcing (figure $23 h 1, h 2$ ), where the cross-sectional plane is characterized by the vorticity concentrations of a single sign. The observations reconfirm that the combined forcing mode is associated with a much better mixing and, furthermore, probably also small-scale mixing.

\subsection{Insight into the three-dimensional flow structure}

To gain insight into the three-dimensional flow structure, we deploy a linear stochastic estimation method to reconstruct the predominant flow structure from the PIV data, captured in the $y-z$ plane at $x / D=0.25$ with a total of 200 images. This technique is introduced in detail by, for example, Adrian \& Moin (1988) and is briefly described below.

Let $q(y, z, t)$ be a velocity component in the cross-plane of $x / D=0.25$ recorded at a constant sampling rate with time step $\Delta t$, i.e. $t_{m}=m \Delta t, m=1,2, \ldots, M$, where $M$ denotes the total number of the PIV snapshots. Use $q^{m}(y, z)=q\left(y, z, t^{m}\right)$ to denote the corresponding snapshots. The mean flow is given by

$$
\bar{q}(y, z)=\frac{1}{M} \sum_{m=1}^{M} q^{m}(y, z) .
$$

The oscillatory actuation response is the corresponding Fourier component of the fluctuation $q^{\prime}=q-\bar{q}$. The cosine and sine contributions read as

$$
\begin{gathered}
q_{1}(y, z)=\frac{2}{M} \sum_{m=1}^{M} \cos \phi^{m}\left[q^{m}(y, z)-\bar{q}(y, z)\right], \\
q_{1}(y, z)=\frac{2}{M} \sum_{m=1}^{M} \sin \phi^{m}\left[q^{m}(y, z)-\bar{q}(y, z)\right], \\
\phi^{m}=2 \pi f_{a} t^{m} .
\end{gathered}
$$

Thus, the periodic flow response may be given by

$$
\widetilde{q}(y, z, t)=\bar{q}(y, z)+q_{1}(y, z) \cos \phi(t)+q_{2}(y, z) \sin \phi(t), \phi=2 \pi f_{a} t .
$$

The residual of this phase-averaged flow $\widetilde{q}$ consists of higher harmonics and an uncorrelated stochastic contribution. Note that the actuation commands $b_{i}(t)$ are the functions of the pointer $(\cos \phi, \sin \phi)$. Hence, the temporal Fourier component optimally represents the actuation response and no flow-intrinsic phase needs to be constructed.

The isosurfaces of reconstructed $\widetilde{V}$ and $\widetilde{W}$ are presented in figure 24 for four control modes. The flow structures of axisymmetric, helical and flapping forcing modes show excellent agreement with the control laws (figure 9). Under axisymmetric forcing (figure 24a1-a2), $\widetilde{V}$ and $\widetilde{W}$ indicate clearly that jet fluids on the two sides of the centreline move either inward or outward simultaneously (figure 24a). Under helical forcing (figure 9h1-h6), the helical motion is evident (figure 24h). For flapping forcing, as indicated by the isosurfaces of $\widetilde{W}$, one sector of fluid moves in one direction for one instant and the adjacent sectors move in the opposite direction 

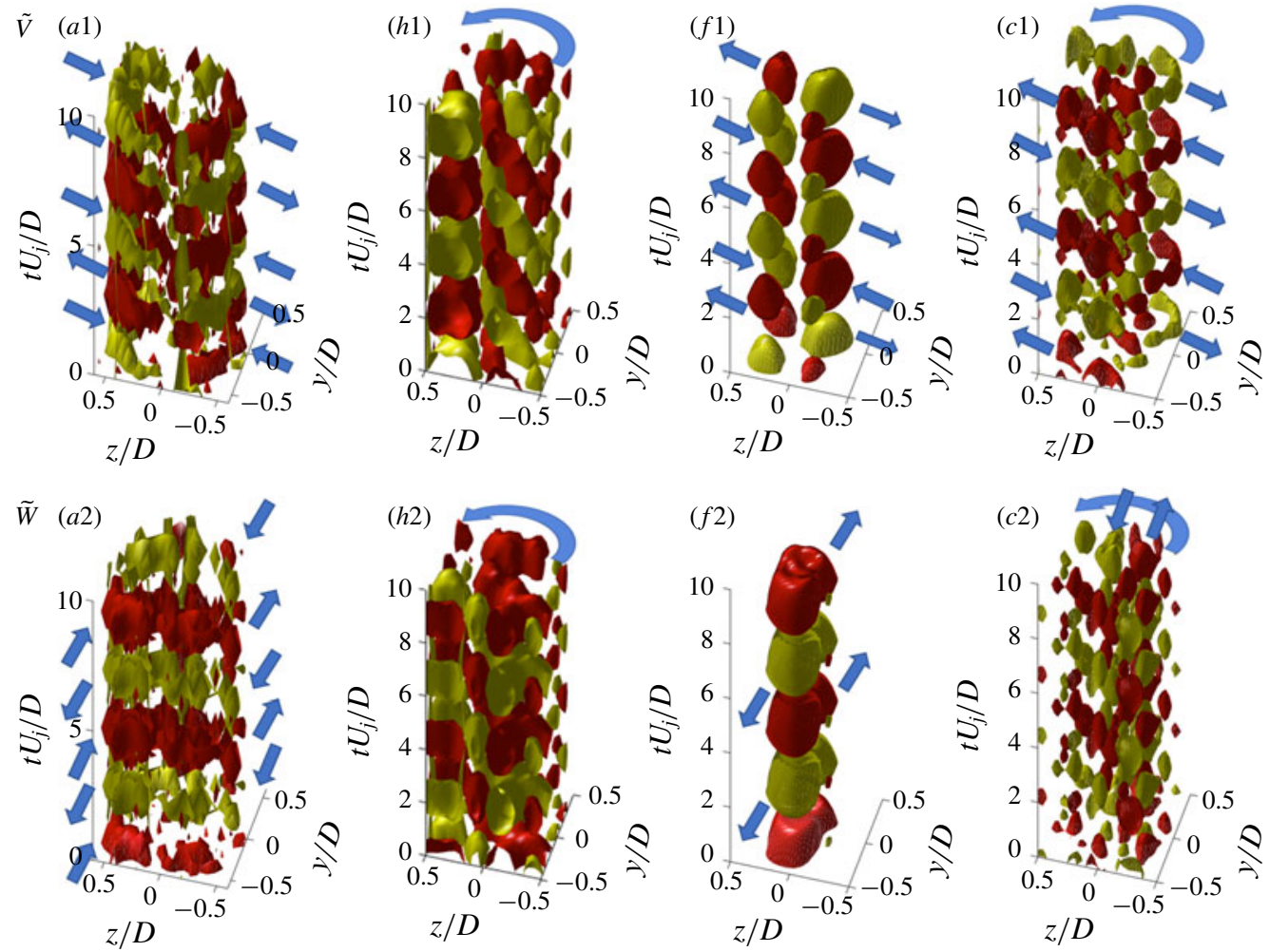

FIGURE 24. Identification of large coherent structures by isosurfaces of phase-averaged velocity for $(a)$ axisymmetric $(h)$ helical $(f)$ flapping $(c)$ combined forcings at $x / D=0.25$. The blue arrow indicates the direction of the jet flow.

(figure 24f2). On the other hand, the isosurfaces of $\widetilde{V}$ is anti-symmetrical about the centreline (figure 24f1). The observations are fully consistent with the characteristics of the flapping motion documented in, for example, Yang \& Zhou (2016). The results provide a validation for the presently reconstructed $\widetilde{V}$ and $\widetilde{W}$. For the combined mode (figure $24 c$ ), the flow structure appears much more complicated. However, the swirling motion is still discernible. Furthermore, the isosurfaces of $\widetilde{V}$ and $\widetilde{W}$ show unequivocally the occurrence of many more small parcels of fluids, suggesting a much better jet mixing than all other flow modes, which is fully consistent with the finding from figure 8 as well as figure $23(c)$.

\section{Conclusions and outlook}

An artificial intelligence (AI) control system has been developed for the control of turbulence. The system consists of a control plant, a sensing unit, an actuation unit and a control or 'thinking' unit. A round jet (control plant) is manipulated to illustrate the potential of this system. Two hot wires are deployed for sensing online the information on the turbulent jet. The control unit deploys a linear genetic programing, and six independent unsteady radial minijets placed around the nozzle prior to the issue of the main jet for executing control laws generated from the control unit. The search space for control laws is extremely large, including the 
minijet number $N$, geometric configuration, frequency $f_{a}$, duty cycle $\alpha$ and phase shift $\varphi_{i j}(i, j=1,2, \ldots, 6)$ between minijets. The target is to maximize the decay rate $K$ of the jet centreline mean velocity, which is correlated with the mixing rate.

It has been demonstrated that the AI control system can learn automatically how to optimize the spatially distributed actuators and thus the turbulent jet for the targeted cost. Like virtually all other control strategies of nonlinear dynamics, AI control solutions do not come with a proof of global optimality. Yet, the results for jet mixing optimization demonstrate several highly desirable features. Firstly, AI control has identified a few typical and well-known control laws, i.e. axisymmetric, helical and flapping forcings, in its learning process in the order of increased control performances, and eventually converged to an unexpected spatio-temporal forcing, referred to as the combined mode, which has never been reported previously. The learning time of 1100 individuals or $2 \mathrm{~h}$ wind-tunnel testing is remarkably short for such a complex solution. It is worth pointing out that the presently developed AI control method may not be suitable for numerical simulation where faster learners, e.g. the reduced-order-model-based models, would be required (once the winning control mechanism is known) to reduce significantly the testing time for $\mathrm{N}-\mathrm{S}$-based simulations. Inspirations may be gained from control studies of a turbulent boundary layer (Sasaki et al. 2020) or turbulent separation (Nair et al. 2019). Secondly, the AI-learned combined mode is reproducible with other initial generations. The control laws may analytically differ but produce almost identical actuation commands. Thirdly, the parameters of the underlying linear genetic programming are taken verbatim from Duriez et al. (2016) and were already proven useful in many other experiments. No sensitive dependence on the parameters has been observed so far and AI control can be expected to yield near-optimal results in its first application to a new plant. Finally, the unique advantage of the AI control over conventional techniques, be open or closed-loop and linear or nonlinear, is its capability to find the apparently global optimum solution when the search space for control laws is extremely large. This advantage will be lost in case of a single actuator involving few control parameters such as frequency and duty cycle (e.g. Fan et al. 2017).

The control landscape is studied by examining the feature vectors of generations $n=1,2,5$ and 11. Several interesting findings are made. Firstly, jet mixing benefits from the increasing asymmetry of forcing in the learning process. Secondly, the feature vectors appear randomly distributed at $n=1$ but evolve gradually to three distinct regions with increasing $n$ (figure 12), characterized by axisymmetric, helical and flapping forcings, respectively. While the region of axisymmetric forcing contracts to one single point, the other two shrink essentially to two curves in the evolution process. Intriguingly, the converged globally optimized control law at $n=11$ takes place in the region of helical forcing which is now populated with either the helical or combined mode. Thirdly, the feature coordinate $\gamma_{1}$ is found to be correlated with the degree of asymmetric forcing, while $\gamma_{2}$ is linked to the number of simultaneously injecting minijets. The best control law is characterized by the largest $\gamma_{2}$ for axisymmetric forcing and largest $\gamma_{1}$ for the other two forcings. However, the globally optimized control law (combined mode) is associated with neither the largest $\gamma_{1}$ nor the largest $\gamma_{2}$.

This combined forcing has produced a novel turbulent flow structure characterized by strong oscillation and swirling motions, along with the generation of mushroomlike structures, all acting to enhance jet mixing. As a result, the combined mode vastly outperforms the well-known optimal benchmark forcings, increasing the entrainment or mixing rate by $54 \%, 47 \%$ and $28 \%$ compared with the axisymmetric, helical 
and flapping forcings, respectively. Extensive measurements are conducted in three orthogonal planes to understand this novel flow structure, which is compared with the flow structures under the benchmark axisymmetric, helical and flapping forcings. It has been found that this flow structure is characterized by a number of features, including the (1) helical motion, (2) three-dimensional oscillating jet column, (3) strong coherent structures, (4) asymmetrical cross-flow distributions of mean and fluctuating velocities, the latter displaying twin-peak behavior in every plane through the $x$-axis, (5) spiral behavior of the swirling jet centre, (6) changing speed of swirling, (7) spectral phase shift by $\pi$ over a considerable frequency band about $f / f_{a}=1$ between two simultaneously captured hot-wire signals placed at $(x / D, y / D)=(1, \pm 0.3)$, and (8) many vorticity concentrations of opposite signs over the entire cross-sectional jet plane, which is distinct from the flow structures under other forcings and suggests better and smaller scale mixing. It is further found that features (1)-(5) resemble those of a precession jet generated by a passive device.

We expect that AI control will be commonly applied to discover the unknown winning nonlinear actuation mechanism of multi-input multi-output flow control experiments in the very foreseeable future. Conventional model-based or modelfree control design may then be deployed to refine and optimize the AI-based actuation mechanism, provided the control law is sufficiently simple. Artificial intelligence control may be improved in numerous aspects. Examples include a human-interpretable control law, an increased learning speed in experiments, robustness against varying operating conditions and the inclusion of prior knowledge and expectations of control laws. One can safely assume that AI will be an essential tool in future turbulence control applications, just as AI is indispensable in robotics now.

\section{Acknowledgements}

Y.Z. wishes to acknowledge support given to him from NSFC through grants 11632006, 91752109 and 91952204. This work is supported by the French National Research Agency (ANR) via the grants ANR-11-IDEX-0003-02 (iCODE), 'ACTIV ROAD' and 'FlowCon', and by the OpenLab Fluidics consortium (Fluidics@poitiers) of PSA Peugeot-Citroë $\mathrm{n}$ and Institute Pprime.

\section{Declaration of interests}

The authors report no conflict of interest.

\section{Appendix A. Control laws}

Artificial intelligence control discovers four typical actuations, as given below. The best individual of the first generation or stage 1 is characterized by an axisymmetric control law:

$$
b_{1}=b_{2}=b_{3}=b_{4}=b_{5}=b_{6}=-0.832+\sin \left(\omega_{a} t+4 / 6 \pi\right) .
$$

Stage 2 starts with the second generation when the AI control discovers a helical forcing:

$$
\begin{gathered}
b_{1}=\sin \left(\omega_{a} t+4 / 6 \pi\right)-0.145, \\
b_{2}=-0.347 \sin \omega_{a} t, \\
b_{3}=\left[\sin \left(\omega_{a} t+8 / 6 \pi\right)+\sin \left(\omega_{a} t+8 / 6 \pi\right)^{2}+\sin \left(\omega_{a} t+2 / 6 \pi\right)^{2}\right] \\
\times \sin \left(\omega_{a} t+8 / 6 \pi\right),
\end{gathered}
$$




$$
\begin{gathered}
b_{4}=2 \sin \left(\omega_{a} t+10 / 6 \pi\right)\left[\left(\sin \left(\omega_{a} t\right)^{2}-\sin \left(\omega_{a} t+2 / 6 \pi\right)\right.\right. \\
\left.\times\left(\sin \left(\omega_{a} t\right)^{2}-\sin \left(\omega_{a} t+2 / 6 \pi\right)\right)\right] \\
b_{5}=1 /\left(-0.347+\sin \omega_{a} t\right)+\sin \omega_{a} t \\
b_{6}=-0.354 \sin \left(\omega_{a} t+8 / 6 \pi\right) .
\end{gathered}
$$

Flapping forcing takes place in stage 3 , starting from the fifth generation:

$$
\begin{aligned}
& b_{1}=b_{2}=b_{3}=-0.811+\sin \left(\omega_{a} t+2 / 6 \pi\right), \\
& b_{4}=b_{5}=b_{6}=-0.782-\sin \left(\omega_{a} t+2 / 6 \pi\right) .
\end{aligned}
$$

The learning process converges to a complex control law in the eleventh generation:

$$
\begin{aligned}
b_{1}= & \left(\left(\sin \left(\omega_{a} t+6 / 6 \pi\right) / \sin \left(\omega_{a} t+4 / 6 \pi\right)\right)^{2} / \sin \omega_{a} t-\sin \omega_{a} t\right) /\left(\sin \omega_{a} t\right)^{4}, \\
b_{2}= & \left(\left(\left(\sin \left(\omega_{a} t+10 / 6 \pi\right) /\left(\sin \omega_{a} t\right)^{2}-1 / \sin \omega_{a} t-\sin \omega_{a} t\right) /\right.\right. \\
& \left.\left.\left(\sin \omega_{a} t\right)^{2}+\sin \omega_{a} t\right) /\left(\sin \omega_{a} t\right)^{3} / \sin \left(\omega_{a} t+10 / 6 \pi\right)-\sin \omega_{a} t\right) /\left(\sin \omega_{a} t\right)^{2} / \\
& \sin \left(\omega_{a} t+8 / 6 \pi\right) /-0.811 /\left(\sin \left(\omega_{a} t+10 / 6 \pi\right) /\left(\sin \omega_{a} t\right)^{2}-\sin \omega_{a} t\right) /\left(\sin \omega_{a} t\right)^{2} / \\
& \sin \left(\omega_{a} t+8 / 6 \pi\right) /-0.811, \\
& b_{3}=-811 /\left(-0.811+\sin \left(\omega_{a} t+2 / 6 \pi\right)+\left(0.482-\sin \left(\omega_{a} t+10 / 6 \pi\right)\right)^{2}\right) / \\
& \left(0.482-\sin \left(\omega_{a} t+10 / 6 \pi\right)\right), \\
& b_{4}=\sin \left(\omega_{a} t+10 / 6 \pi\right)-2 \sin \left(\omega_{a} t+2 / 6 \pi\right)-0.223+\left(\sin \left(\omega_{a} t+2 / 6 \pi\right)\right. \\
b_{5}= & \left(\left(\sin \left(\omega_{a} t+10 / 6 \pi\right) /\left(\sin \omega_{a} t\right)^{2}-\sin \omega_{a} t\right) /\left(\sin \omega_{a} t\right)^{2}+\sin \omega_{a} t\right) / \\
& \left(-0.782+\sin \left(\omega_{a} t+2 / 6 \pi\right)-\sin \left(\omega_{a} t+10 / 6 \pi\right) /\left(\sin \omega_{a} t\right)^{2} / \sin \left(\omega_{a} t+8 / 6 \pi\right)\right. \\
& +\left(\sin \left(\omega_{a} t+10 / 6 \pi\right) /\left(\sin \omega_{a} t\right)^{2}-\sin \omega_{a} t\right) /\left(\sin \omega_{a} t\right)^{2} /\left(-0.782+\sin \left(\omega_{a} t+2 / 6 \pi\right)\right. \\
& \left.+\left(-0.782+\sin \left(\omega_{a} t+2 / 6 \pi\right)\right)^{2}\right) / \\
& \left(\left(\sin \left(\omega_{a} t+10 / 6 \pi\right) /\left(\sin \omega_{a} t\right)^{2}-\sin \omega_{a} t\right) /\left(\sin \omega_{a} t\right)^{2}\right. \\
& \left.+\sin \omega_{a} t\right) /\left(-0.782+\sin \left(\omega_{a} t+2 / 6 \pi\right)-\sin \left(\omega_{a} t+10 / 6 \pi\right) /\left(\sin \omega_{a} t\right)^{2}\right) / \\
& \left(\sin \left(\omega_{a} t+8 / 6 \pi\right)+\sin \left(\omega_{a} t+10 / 6 \pi\right) /\left(\sin \omega_{a} t\right)^{2}-\sin \omega_{a} t\right) /\left(\sin \omega_{a} t\right)^{2} / \\
& \left(-0.782+\sin \left(\omega_{a} t+2 / 6 \pi\right)-\sin \left(\omega_{a} t+10 / 6 \pi\right)\right) /\left(\sin \left(\omega_{a} t+8 / 6 \pi\right)\right. \\
& \left.+\left(\sin \left(\omega_{a} t+10 / 6 \pi\right) /\left(\sin \omega_{a} t\right)^{2}-\sin \omega_{a} t\right) /\left(\sin \omega_{a} t\right)^{2}\right)+\left(\left(\left(\sin \left(\omega_{a} t+10 / 6 \pi\right) /\right.\right.\right. \\
& \left.\left.\left(\sin \omega_{a} t\right)^{2}-\sin \omega_{a} t\right) /\left(\sin \omega_{a} t\right)^{2}+\sin \omega_{a} t\right) /\left(\left(-0.782+\sin \left(\omega_{a} t+2 / 6 \pi\right)\right.\right. \\
& \left.-\sin \left(\omega_{a} t+10 / 6 \pi\right) /\left(\sin \omega_{a} t\right)^{2}\right) / \sin \left(\omega_{a} t+8 / 6 \pi\right)+\left(\sin \left(\omega_{a} t+10 / 6 \pi\right) /\right. \\
& \left.\left.\left.\left.\left(\sin \omega_{a} t\right)^{2}-\sin \omega_{a} t\right) /\left(\sin \omega_{a} t\right)^{2}\right)\right)\right), \\
& \quad b_{6}=2 \sin \left(\omega_{a} t+10 / 6 \pi\right)-\sin \left(\omega_{a} t+6 / 6 \pi\right) .
\end{aligned}
$$

\section{REFERENCES}

ADRIAN, R. J. \& MoIN, P. 1988 Stochastic estimation of organized turbulent structure: homogeneous shear flow. J. Fluid Mech. 190, 531-559.

Alkislar, M. B., Krothapalli, A. \& Butler, G. W. 2007 The effect of streamwise vortices on the aeroacoustics of a Mach 0.9 jet. J. Fluid Mech. 578, 139-169. 
Andreopoulos, J. \& Rodi, W. 1984 Experimental investigation of jets in a crossflow. J. Fluid Mech. 138, 93-127.

Bradbury, L. J. S. \& Khadem, A. H. 1975 The distortion of a jet by tabs. J. Fluid Mech. 70 (4), 801-813.

Broze, G. \& Hussain, F. 1994 Nonlinear dynamics of forced transitional jets: periodic and chaotic attractors. J. Fluid Mech. 263, 93-132.

Carlos, B. S. \& Olivier, M. 2002 Vortex control of bifurcating jets: a numerical study. Phys. Fluids 14 (11), 3798-3819.

Cox, T. \& Cox, M. 2000 Multidimensional Scaling. Chapman \& Hall.

Crow, S. C. \& Champagne, F. H. 1971 Orderly structure in jet turbulence. J. Fluid Mech. 48 (3), 547-591.

DAVIS, M. R. 1982 Variable control of jet decay. AIAA J. 20, 606-609.

Delaunay, B. 1934 Sur la sphère vide. a la mémoire de georges vorono. Bull. Acad. Sci. URSS. Classe des sciences mathématiques et naturelles 6, 793-800.

Dracopoulos, D. C. 1997 Evolutionary Learning Algorithms for Neural Adaptive Control. Springer.

Duriez, T., BRUnton, S. \& NoACK, B. R. 2016 Machine Learning Control - Taming Nonlinear Dynamics and Turbulence. Springer.

FAn, D. W., Wu, Z., YANG, H., LI, J. D. \& Zhou, Y. 2017 Modified extremum-seeking closed-loop system for jet mixing enhancement. AIAA J. 55 (11), 3891-3902.

Garnaud, X., Lesshafft, L., Schmid, P. J. \& Huerre, P. 2013 The preferred mode of incompressible jets: linear frequency response analysis. J. Fluid Mech. 716, 189-202.

Gautier, N., Aider, J. L., Duriez, T., Noack, B. R., Segond, M. \& Abel, M. 2015 Closed-loop separation control using machine learning. J. Fluid Mech. 770, 442-457.

Goldschmidt, V. W. \& Bradshaw, P. 1973 Flapping of a plane jet. Phys. Fluids 16 (3), 354-355.

Gutmark, E. \& Ho, C. M. 1983 Preferred modes and the spreading rates of jets. Phys. Fluids 26 (10), 2932-2938.

Hilgers, A. \& Boersma, B. J. 2001 Optimization of turbulent jet mixing. Fluid Dyn. Res. 29, $345-368$.

Ho, C. M. \& Huerre, P. 1984 Perturbed free shear layers. Annu. Rev. Fluid Mech. 16, 365-422.

HuAnG, J. M. \& HsiaO, F. B. 1999 On the mode development in the developing region of a plane jet. Phys. Fluids 11 (7), 1847-1857.

Husain, H. S. \& Hussain, A. K. M. F. 1983 Controlled excitation of elliptic jets. Phys. Fluids 26 (10), 2763-2766.

Hussain, F. \& Husain, H. S. 1989 Elliptic jets. Part 1. Characteristics of unexcited and excited jets. J. Fluid Mech. 208, 257-320.

Johari, H., Pachecotougas, M. \& Hermanson, J. 1999 Penetration and mixing of fully modulated turbulent jets in crossflow. AIAA J. 37 (7), 842-850.

Juvet, P. J. D. 1987 Control of high Reynolds number round jets. PhD thesis, Stanford Unversity, Stanford, CA.

Kaiser, E., Noack, B. R., Spohn, A., Cattafesta, L. N. \& Morzyński, M. 2017 Cluster-based control of a separating flow over a smoothly contoured ramp. Theor. Comput. Fluid Dyn. 31 (5), 579-593.

Kim, J. \& Bewley, T. R. 2007 A linear systems approach to flow control. Annu. Rev. Fluid Mech. 39 (1), 383-417.

Koch, C. R., Mungal, M. G., Reynolds, W. C. \& Powell, J. D. 1989 Helical modes in an acoustically excited round air jet. Phys. Fluids 1 (9), 1443-1443.

Koenig, M., Sasaki, K., Cavalieri, A. V. G., Jordan, P. \& Gervais, Y. 2016 Jet-noise control by fluidic injection from a rotating plug: linear and nonlinear soun-source mechanisms. J. Fluid Mech. 788, 358-380.

Koumoutsakos, P., Freund, J. \& Parekh, D. 2001 Evolution strategies for automatic optimization of jet mixing. AIAA J. 39 (5), 967-969.

LeE, M. \& ReYnolds, W. C. 1985 Bifurcating and blooming jets. Dep. Mech. Eng. Thermosci. Div. Rep. TF-22, Stanford University. 
Li, R., Noack, B. R., Cordier, L., Borée, J., Kaiser, E. \& Harambat, F. 2017 Linear genetic programming control for strongly nonlinear dynamics with frequency crosstalk. Arch. Mech. 70 (6), 505-534.

LIEPMANN, D. \& GHARIB, M. 1992 The role of streamwise vorticity in the near-field entrainment of round jets. J. Fluid Mech. 245, 643-668.

Longmire, E. K. \& DuOng, L. H. 1996 Bifurcating jets generated with stepped and sawtooth nozzles. Phys. Fluids 8 (4), 978-992.

Malmstrom, T. G., Kirkpatrick, A. T., Christensen, B. \& Knappmiller, K. D. 1997 Centreline velocity decay measurements in low-velocity axisymmetric jets. J. Fluid Mech. 346, 363-377.

M'closkey, R. T., King, J. M. \& Cortelezzi, L. 2002 The actively controlled jet in crossflow. J. Fluid Mech. 452, 325-335.

Mi, J. \& NATHAN, G. J. 2005 Statistical analysis of the velocity field in a mechanical precessing jet flow. Phys. Fluids 17 (1), 015102.

Mi, J., Nobes, D. S. \& Nathan, G. J. 2001 Influence of jet exit conditions on the passive scalar field of an axisymmetric free jet. J. Fluid Mech. 432, 91-125.

MI, J., XU, M. \& ZHOU, T. 2013 Reynolds number influence on statistical behaviors of turbulence in a circular free jet. Phys. Fluids 25 (7), 075101.

Nair, A. G., Yeh, C. A., Kaiser, E., Noack, B. R., Brunton, S. L. \& TAira, K. 2019 Cluster-based feedback control of turbulent post-stall separated flows. J. Fluid Mech. 875, 345-375.

Nathan, G. J., Hill, S. J. \& Luxton, R. E. 1998 An axisymmetric nozzle to generate jet precession. J. Fluid Mech. 370, 347-380.

NoACK, B. R. 2019 Closed-loop turbulence control - from human to machine learning (and retour). In Proceedings of the 4th Symposium on Fluid Structure-Sound Interactions and Control (FSSIC), Tokyo, Japan (ed. Y. Zhou, M. Kimura, G. Peng, A. D. Lucey \& L. Hung), pp. 1-10. Springer.

Parezanović, V., Cordier, L., Spohn, A., Duriez, T., Noack, B. R., Bonnet, J. P., Segond, M., Abel, M. \& BRunton, S. L. 2016 Frequency selection by feedback control in a turbulent shear flow. J. Fluid Mech. 797, 247-283.

Paschereit, C. O., Wygnanski, I. \& Fiedler, H. E. 1995 Experimental investigation of subharmonic resonance in an axisymmetric jet. J. Fluid Mech. 283, 365-407.

Perumal, A. K. \& Zhou, Y. 2018 Parametric study and scaling of jet manipulation using an unsteady minijet. J. Fluid Mech. 848, 592-630.

Raman, G., Hailye, M. \& Rice, E. J. 1993 Flip-flop jet nozzle extended to supersonic flows. AIAA J. 31 (6), 1028-1035.

ReChenberg, I. 1973 Evolutionsstrategie: Optimierung Technischer Systeme nach Prinzipien der Biologischen Evolution. Frommann-Holzboog.

ReEder, M. F. \& SAmimy, M. 1996 The evolution of a jet with vortex-generating tabs: real-time visualization and quantitative measurements. J. Fluid Mech. 311, 73-118.

Reynolds, W. C., Parekh, D. E., Juvet, P. J. D. \& Lee, M. J. D. 2003 Bifurcating and blooming jets. Аnпи. Rev. Fluid Mech. 35, 295-315.

SAIlor, D. J., Rohli, D. J. \& FU, Q. 1999 Effect of variable duty cycle flow pulsations on heat transfer enhancement for an impinging air jet. Intl J. Heat Fluid Flow 20 (6), 574-580.

Samimy, M., Kim, J. H., Kastner, J., Adamovich, I. \& UtKin, Y. 2007 Active control of highspeed and high-Reynolds-number jets using plasma actuators. J. Fluid Mech. 578, 305-330.

Sasaki, K., Morra, P., Cavalieri, A. V. G., Hanifi, A. \& Henningson, D. S. 2020 On the role of actuation for the control of streaky structures in boundary layers. J. Fluid Mech. 883, A34.

Sasaki, K., Piantanida, S., Cavalieri, A. V. G. \& Jordan, P. 2017 Real-time modelling of wavepackets in turbulent jets. J. Fluid Mech. 821, 458-481.

Sasaki, K., Tissot, G., Cavalieri, A. V. G., Silvestre, F. J., Jordan, P. \& Biau, D. 2018 Closed-loop control of a free shear flow: a framework using the parabolized stability equations. Theor. Comput. Fluid Dyn. 32 (6), 765-788. 
Seidel, J., Pappert, C., New, T. H. \& Tsai, H. M. 2005 Effects of multiple radial blowing around a circular jet. AIAA Paper 2005-866.

Sipp, D., Marquet, O., Meliga, P. \& Barbagallo, A. 2010 Dynamics and control of global instabilities in open-flows: a linearized approach. Appl. Mech. Rev. 63 (3), 030801.

TAmburello, D. A. \& Amitay, M. 2007 Three-dimensional interactions of a free jet with a perpendicular synthetic jet. J. Turbul. 8, N38.

Tinney, C. E., Coiffet, F., Delville, J., Hall, A. M., Jordan, P. \& Glauser, M. N. 2006 On spectral linear stochastic estimation. Exp. Fluids 41 (5), 763-775.

Todde, V., Spazzini, P. G. \& SAndberG, M. 2009 Experimental analysis of low-Reynolds number free jets. Exp. Fluids 47 (2), 279-294.

TYliszCZAK, A. 2018 Parametric study of multi-armed jets. Intl J. Heat Fluid Flow 73, 82-100.

TYliszcZAK, A. \& GeURTS, B. J. 2015 Controlled mixing enhancement in turbulent rectangular jets responding to periodically forced inflow conditions. J. Turbul. 16 (8), 742-771.

Vlasov, E. V. \& GinevskiI, A. S. 1973 Generation and suppression of turbulence in an axisymmetric turbulent jet under an acoustic effect. Fluid Dyn. 8 (6), 881-885.

Wiltse, J. M. \& Glezer, A. 1993 Manipulation of free shear flows using piezoelectric actuators. J. Fluid Mech. 249, 261-285.

Wong, C. Y., Lanspeary, P. V., Nathan, G. J., Kelso, R. M. \& O’Doherty, T. 2003 Phaseaveraged velocity in a fluidic precessing jet nozzle and in its near external field. Exp. Therm. Fluid Sci. 27 (5), 515-524.

Wong, C. Y., Nathan, G. J. \& O'Doherty, T. 2004 The effect of initial conditions on the exit flow from a fluidic precessing jet nozzle. Exp. Fluids 36 (1), 70-81.

Wu, Z., FAN, D., Zhou, Y., LI, R. \& NOACK, B. R. $2018 a$ Jet mixing enhancement using machine learning control. Exp. Fluids 59 (8), 131.

Wu, Z., Wong, C. W. \& ZHOU, Y. $2018 b$ Dual-input/single-output extremum-seeking system for jet control. AIAA J. 56 (4), 1463-1471.

YANG, H. 2017 Study of active jet control using unsteady minijets (translated). PhD thesis, Harbin Institute of Technology, China.

YANG, H. \& ZhOU, Y. 2016 Axisymmetric jet manipulated using two unsteady minijets. J. Fluid Mech. 808, 362-396.

YANG, H., ZhOU, Y., So, R. M. \& LiU, Y. 2016 Turbulent jet manipulation using two unsteady azimuthally separated radial minijets. Proc. R. Soc. Lond. A 472 (2191), 20160417.

ZAMAN, K. \& Hussain, A. 1981 Turbulence suppression in free shear flows by controlled excitation. J. Fluid Mech. 103, 133-159.

ZAMAN, K. B. M. Q. 1996 Axis switching and spreading of an asymmetric jet - role of vorticity dynamics. J. Fluid Mech. 316, 1-27.

Zaman, K. B. M. Q., Reeder, M. F. \& SAmimy, M. 1994 Control of an axisymmetric jet using vortex generators. Phys. Fluids 6 (2), 778-793.

ZhANG, S. \& TuRner, J. T. 2016 On the development of a turbulent jet subjected to aerodynamic excitation in the helical mode. Exp. Therm. Fluid Sci. 78, 278-291.

Zhou, Y., Du, C., Mi, J. \& WANG, X. W. 2012 Turbulent round jet control using two steady minijets. AIAA J. 50 (3), 736-740.

Zhou, Y., Zhang, H. J. \& YiU, M. W. 2002 The turbulent wake of two side-by-side circular cylinders. J. Fluid Mech. 458, 303-332. 